\title{
A multiwavelength strong lensing analysis of baryons and dark matter in the dynamically active cluster AC 114
}

\author{
M. Sereno ${ }^{1,2,3}$, M. Lubini ${ }^{1}$, and Ph. Jetzer ${ }^{1}$ \\ 1 Institut für Theoretische Physik, Universität Zürich, Winterthurerstrasse 190, 8057 Zürich, Switzerland \\ e-mail: mauro.sereno@polito.it \\ 2 Dipartimento di Fisica, Politecnico di Torino, Corso Duca degli Abruzzi 24, 10129 Torino, Italy \\ 3 INFN, Sezione di Torino, via Pietro Giuria 1, 10125 Torino, Italy
}

Received 10 December 2009 / Accepted 20 April 2010

\section{ABSTRACT}

\begin{abstract}
Context. Strong lensing studies can provide detailed mass maps of the inner regions even in dynamically active galaxy clusters. Aims. We illustrate the important role of a proper modelling of the intracluster medium, i.e., the main baryonic component. We demonstrate that the addition of a new contribution accounting for the gas can increase the statistical significance of the lensing model.

Methods. We propose a parametric method for strong lensing analyses that exploits multiwavelength observations. The mass model accounts for cluster-sized dark matter halos, galaxies (whose stellar mass can be obtained from optical analyses), and the intracluster medium. The gas distribution is fitted to lensing data exploiting prior knowledge from X-ray observations. This gives an unbiased insight into each matter component and allows us to study the dynamical status of a cluster. The method was applied to AC 114, an irregular X-ray cluster.

Results. We find positive evidence of dynamical activity, the dark matter distribution being shifted and rotated with respect to the gas. On the other hand, the dark matter follows the galaxy density in terms of both shape and orientation, illustrating the collisionless nature of dark matter. The inner region $(<250 \mathrm{kpc})$ is underluminous in optical bands, whereas the gas fraction $(\sim 20 \pm 5 \%)$ slightly exceeds typical values. Evidence of lensing and X-ray suggests that the cluster develops in the plane of the sky and is not affected by the lensing over-concentration bias. Despite the dynamical activity, the matter distribution seems to agree with predictions of $N$-body simulations. An universal cusped profile provides a good description of either the overall or the dark matter distribution, whereas theoretical scaling relations seem to be accurately fitted.
\end{abstract}

Key words. galaxies: clusters: general - X-rays: galaxies: clusters - gravitational lensing: strong

\section{Introduction}

Understanding the formation and evolution of galaxy clusters is an open problem in modern astronomy. On the theoretical side, $N$-body simulations are now able to make detailed statistical predictions on dark matter (DM) halo properties (Navarro et al. 1997; Bullock et al. 2001; Diemand et al. 2004; Duffy et al. 2008). On the observational side, multiwavelength observations from the radio to the optical bands to X-ray observations of galaxy clusters can provide deep insight into real features (Clowe et al. 2004; De Filippis et al. 2005; Smith et al. 2005; Hicks et al. 2006). Results are impressive on both sides, but additional work is still required. Large numerical simulations still cannot efficiently incorporate gas physics, whereas combining multiwavelength data sets can be misleading if the employed hypotheses (hydrostatic and/or dynamical equilibrium, spherical symmetry, just to list a couple of very common ones) do not hold. Areas of disagreement between predictions and measurements therefore persist.

We consider a way of exploiting multiwavelength data sets in strong lensing data analyses. Strong lensing modelling can give detailed maps of the inner regions of galaxy clusters without relying on hypotheses about equilibrium and is negligibly affected by projection effects caused by large-scale fields or aligned structures. However, massive lensing clusters represent a biased sample for statistical studies (Hennawi et al. 2007;
Oguri \& Blandford 2009). Multiwavelength analyses of lensing galaxy clusters have been exploited following different approaches. Smith et al. (2005) compared X-ray and strong lensing maps of intermediate redshift clusters to infer equilibrium criteria. Detailed lensing features can reveal dynamical activity even in apparently relaxed clusters (Miranda et al. 2008). Investigations of the bullet cluster showed that dark matter follows the collisionless galaxies, whereas the gas is stripped away by mergers (Clowe et al. 2004). Comparison of snapshots of active clusters taken with weak lensing, X-ray surface brightness, or galaxy luminosity revealed the relative displacement of the different components at different stages of merging (Okabe \& Umetsu 2008).

The usual way to differentiate dark matter from baryons in lensing analyses involves first obtaining a map of the total matter distribution fitting the lensing features and then subtracting the gas contribution as inferred from X-ray observations (Bradač et al. 2008). The total mass map can be obtained with either parametric models in which the contribution from cluster-sized DM halos can be considered with the main galactic DM halos (Natarajan et al. 1998; Limousin et al. 2008) or non-parametric analyses, where dark matter meso-structures and galactic contributions are seen as deviations from smooth-averaged profiles (Saha et al. 2007). Mass in stars and stellar remnants is estimated from galaxy luminosity assuming suitable stellar mass to 
light ratios. These approaches have obvious merits but also some unavoidable shortcomings.

Collisionless matter and gas are displaced in dynamical active clusters. Furthermore, gas and dark matter profiles usually have different slopes in relaxed clusters. Since the gas follows the potential, its distribution is usually rounder than dark matter, so that even if the intracluster medium (ICM) and the dark matter are intrinsically aligned their projected masses on the sky have different orientations and ellipticities (Stark 1977). These features can be missed by usual approaches since the total matter profile may no fully account for the properties of each component. In fact, the number of model parameters may indeed be insufficient to reproduce all the details, whereas in non-parametric approaches the fitting procedure is weighted to favour smooth distributions against clumpy ones, a procedure that may remove small scale details such as gas and dark matter that are off-centre by few arcseconds.

We take an additional step by exploiting a parametric model that has three kind of components: cluster-sized dark matter halos; galaxy-sized (dark plus stellar) matter halos; and clustersized gas distribution. In our approach, the ICM distribution is embedded in the strong lensing modelling from the very beginning to avoid unpleasant biases. To reduce the total number of parameters, the X-ray surface brightness data are fully exploited so that the gas contribution is fixed within the observational uncertainties. This allows us to constrain the mass model using X-ray data without relying on the assumption of hydrostatic equilibrium. As far as the stellar component is concerned, we follow the usual approach: total galaxy masses (DM plus baryons) are derived using the lensing fitting procedure, whereas the stellar contribution is inferred from luminosity. We recognise that the main advantage of this approach is that we are able to infer directly the dark matter mass. This is the component most accurately (and under some points of view, the only one) constrained in numerical simulations, so that our novel approach aids the comparison with their theoretical predictions. Furthermore, we will be able to compare the gas distribution with the dark matter, which is an obvious improvement to the usual way of comparing total projected mass distributions with surface brightness maps.

We apply our method to the cluster AC 114, which exhibits a prototypical example of the Butcher-Oemler effect with a higher fraction of blue, late-type galaxies than in lower redshift clusters, rising to $60 \%$ outside the core region (Couch et al. 1998). The fraction of interacting galaxies $(\sim 12 \%)$ is also high (Couch et al. 1998). Krick \& Bernstein (2007) classified AC 114 as a Bautz-Morgan type II-III cluster, which indicates that it has a young dynamical age. The cluster is elongated significantly in the southeast-northwest direction (Couch et al. 2001) (see Fig. 1). There are two main reasons for studying this cluster. First, the core region of AC 114 is rich in multiple images, allowing a very detailed analysis. Redshift contrast between multiply lensed sources can provide a good measurement of the enclosed mass at two different radii, thus providing a good estimate of the mass profile in-between (Saha \& Read 2009). The same kind of information can be obtained by also combining strong and weak lensing data, but multiply lensed sources allow us to consistently derive the profile slope without mixing systematics from different methods. Furthermore, there are several images very near the cluster centre that allow us to accurately determine the radial slope of the matter distribution in the very inner regions. Despite the abundance of data, AC 114 has been the target of only a couple lensing investigations. In the first one by Natarajan et al. (1998), which was later improved by

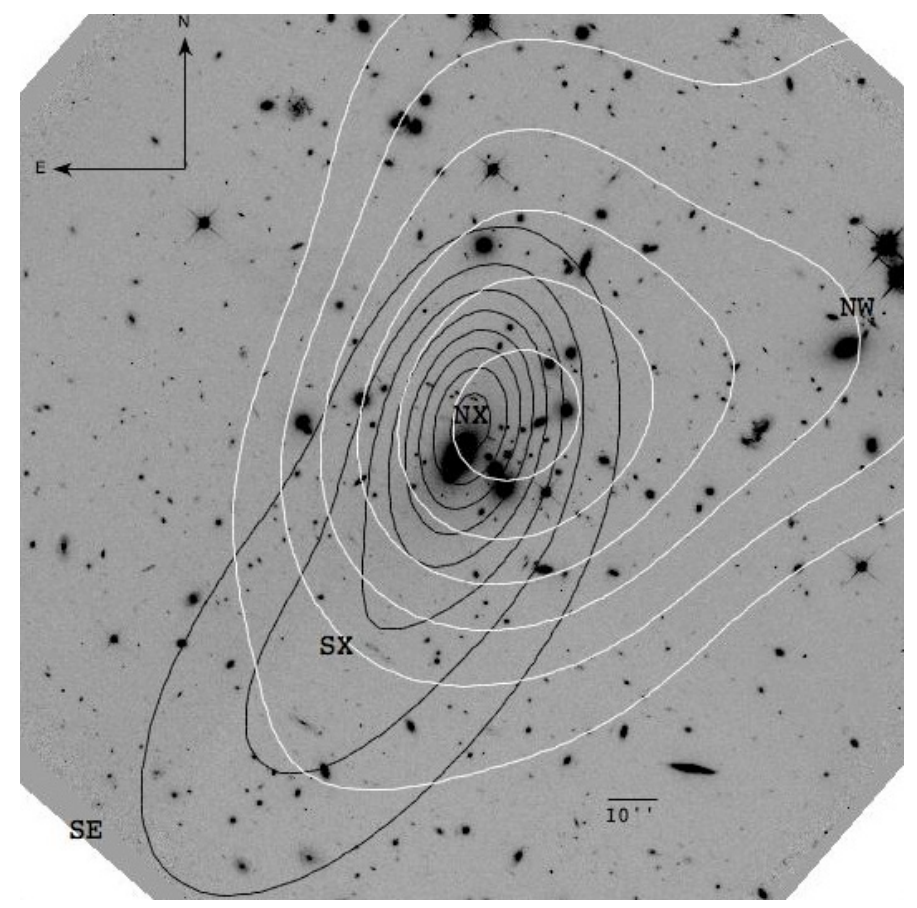

Fig. 1. Grey-scale archive HST/ACS image ( $F 850$ band) of the core of AC 114. North is up and east is to the left. The field covers $3^{\prime} \times 3^{\prime}$. Overlaid are the linearly spaced adaptively-smoothed Chandra surface brightness X-ray inner (black) contours and the inner light (white) contours. Light isophotes were obtained by smoothing the luminosity map with a Gaussian kernel with a dispersion of $30^{\prime \prime}$. NX and SX are the centroids of the main X-ray clump and the southern tail, respectively; NW and SE locate likely mass clumps.

Campusano et al. (2001), weak lensing constraints were also used and the mass modelling, which was inspired by the optical galaxy distribution, considered a main clump and two additional cluster substructures (see Appendix B). The second lensing analysis, inspired by the X-ray images, associated each of the two X-ray emitting regions with a dark matter clump separately in hydrostatic equilibrium (De Filippis et al. 2004). Both approaches reproduced the image positions with an accuracy of $\gtrsim 1^{\prime \prime}$, but neither used all of the image systems with confirmed spectroscopic redshifts. There is therefore room for substantial improvement.

Second, AC 114 exhibits significant evidence of being an ongoing merging. We study in detail the dark matter distribution in a dynamical active cluster, constraining at the same time the properties of the dark matter and the evolution of this interesting cluster. The lensing analysis of the cluster will allow us to compare observations with theoretical predictions.

The paper is as follows. In Sect. 2 we discuss the galaxy distribution and present the measurements of luminosity and number densities. We also consider the stellar mass inferred from the measured luminosity. Section 3 describes our dynamics analysis in which we obtain an updated estimate of the cluster mass that is later compared to the lensing results. In Sect. 4, we review literature results about the X-ray observations of the cluster. Section 5 and 6 are devoted to the lensing analysis. In Sect. 5, we review the optical data and the parametric models employed; in Sect. 6, we present our statistical investigation. Section 7 lists the results obtained with our multiwavelength approach, whereas Sect. 8 discusses some results in the context of theoretical expectations. Section 9 discusses our final considerations. In Appendix A, we detail our procedure to estimate the galaxy velocity dispersion 
M. Sereno et al.: Multiwavelength lensing in AC 114

Table 1. Properties of the galaxy distributions, modelled as $\beta$ profiles, within circular regions of outer radius $\theta_{\max }$.

\begin{tabular}{lccccccc}
\hline \hline Density & $\begin{array}{c}\theta_{\max } \\
\left({ }^{\prime \prime}\right)\end{array}$ & $\beta$ & $\begin{array}{c}\theta_{\mathrm{c}} \\
\left({ }^{\prime \prime}\right)\end{array}$ & $\begin{array}{c}\theta_{1,0} \\
\left({ }^{\prime \prime}\right)\end{array}$ & $\begin{array}{c}\theta_{2,0} \\
\left({ }^{\prime \prime}\right)\end{array}$ & $\epsilon$ & $\begin{array}{c}\theta_{\epsilon} \\
(\mathrm{deg})\end{array}$ \\
\hline Number & 60 & $1.2_{-0.4}^{+0.7}$ & $50_{-30}^{+30}$ & $10_{-4}^{+3}$ & $6_{-4}^{+3}$ & $0.25_{-0.11}^{+0.12}$ & $-40_{-20}^{+20}$ \\
& 120 & $1.07_{-0.15}^{+0.30}$ & $140_{-30}^{+20}$ & $10_{-3}^{+4}$ & $4_{-4}^{+4}$ & $0.53_{-0.04}^{+0.03}$ & $-41_{-3}^{+3}$ \\
\hline Luminosity & 60 & $1.6_{-0.5}^{+2.3}$ & $36_{-13}^{+50}$ & $7_{-3}^{+3}$ & $7_{-5}^{+3}$ & $0.22_{-0.15}^{+0.10}$ & $-30_{-30}^{+30}$ \\
& 120 & $0.86_{-0.10}^{+0.60}$ & $42_{-20}^{+70}$ & $13_{-5}^{+5}$ & $11_{-6}^{+6}$ & $0.42_{-0.08}^{+0.06}$ & $-26_{-10}^{+9}$ \\
\hline Stellar mass & 60 & $1.6_{-0.4}^{+1.5}$ & $36_{-14}^{+30}$ & $8_{-4}^{+3}$ & $6_{-4}^{+3}$ & $0.24_{-0.10}^{+0.10}$ & $-30_{-30}^{+20}$ \\
& 120 & $0.96_{-0.12}^{+0.17}$ & $36_{-14}^{+20}$ & $10_{-5}^{+5}$ & $9_{-5}^{+5}$ & $0.39_{-0.10}^{+0.07}$ & $-32_{-12}^{+12}$ \\
\hline
\end{tabular}

Notes. Angles are measured north over east.

$\sigma_{\text {los }}$. Appendix B is devoted to an analysis of substructures based on classical optical methods. Projection effects are dealt with in Appendix C. Throughout the paper, we assume a $\Lambda$ CDM cosmology with density parameters $\Omega_{\mathrm{M}}=0.3$ and $\Omega_{\Lambda}=0.7$, and a Hubble constant $\mathrm{H}_{0}=100 h \mathrm{~km} \mathrm{~s}^{-1} \mathrm{Mpc}^{-1}$, where $h=0.7$. This implies a linear scale of $3.22 \mathrm{kpc} / h$ per arcsec at the cluster redshift $z=0.315$. We assume the reference mass and radius of the cluster to be $M_{200}$ and $r_{200}$, i.e., the mass and the radius containing an overdensity of 200 times the critical one. We quote uncertainties at the $68.3 \%$ confidence level.

\section{Galaxy distribution}

The galaxy catalogue we made use of is taken from Couch et al. (1998, see their table 4), who morphologically classified galaxies recorded on images taken with WFPC2 at HST down to $R_{702}=23.00$. The galaxy distribution in the inner regions is quite irregular, substructures being detected with different methods, see Appendix B.

Here we analyse the galaxy density distribution by considering either the luminosity or the number density. Our method is as follows. We first smooth the spiky density distribution by convolving with a Gaussian kernel whose fixed width is based on the mean distance between galaxies in the region of interest. The characteristics of the surface distribution are then obtained by considering a sample of maps generated by resampling the data of the original distribution. This takes into account the finite size error. For each map, we perform a parametric fit with Poisson weights to an elliptical density distribution. Parameter central values and confidence intervals are finally obtained by considering median and quantile ranges of the final population of the best-fit model parameters. Throughout the paper, we consider a coordinate system in the plane of the sky, $\left\{\theta_{1}, \theta_{2}\right\}$, centred on the BCG galaxy and aligned with the equatorial system with increasing numbers to both the west and north of the central galaxy. As a surface density model, we assume a projected King-like $\beta$ distribution

$\Sigma=\Sigma_{0}\left[1+\left(\theta_{\mathrm{ell}} / \theta_{\mathrm{P}}\right)^{2}\right]^{(1-3 \beta) / 2}+\Sigma_{\mathrm{B}}$,

where $\theta_{\text {ell }}$ is a projected elliptical radius that measures the major axis length of concentric ellipses in the plane of the sky centred on $\left\{\theta_{1,0}, \theta_{2,0}\right\}$, of ellipticity $\epsilon$, defined as 1 minus the ellipse axial ratio, and orientation angle $\theta_{\epsilon}$ (measured north over east); $\theta_{\mathrm{P}}$ is the projected core radius, $\beta$ parameterizes the slope and $\Sigma_{\mathrm{B}}$ is the constant background. Each parameter in Eq. (1) is allowed to vary in the fitting procedure, and flat priors are used. We consider two circular regions on the sky of external radius $\theta_{\max }=60^{\prime \prime}$ and $\theta_{\max }=120^{\prime \prime}$, containing 104 and 329 galaxies, respectively. For the dispersion of the Gaussian kernel, we used $10^{\prime \prime}$ and 12", respectively. Results are listed in Table 1. The central BCG galaxy is slightly shifted from the luminosity centroid, located northwest, but the statistical significance of this displacement is low. We identify a significant southeastnorthwest elongation. Differences in shape between the luminosity and number density maps are caused by the relative high abundance of late-type galaxies outside the core region. Both the ellipticity and orientation of the luminosity distribution within $2^{\prime}$ strictly follow the ellipticity parameters of the cD galaxy, which we estimated using the ellipse task in the IRAF package to be $\left\{\epsilon, \theta_{\epsilon}\right\}=\{0.42 \pm 0.05,(-32.8 \pm 0.5) \mathrm{deg}\}$.

\subsection{Stellar mass}

The baryonic contribution of stars and stellar remnants can be estimated by converting galaxy luminosities into stellar masses. We convert $R_{702}$ into infrared $K$ luminosity, which is less sensitive to ongoing star formation and is a more reliable tracer of the stellar mass distribution, mostly following Smith et al. (2002, 2005). As a first step, we corrected $R_{702}$ photometry reported in the SExtractor catalogue of Couch et al. (1998) for an overestimate of the background as discussed in Smith et al. (2005) and then converted $R_{702}$ photometry to Cousin $R$, using suitable corrections per morphological type (Smith et al. 2002). We then obtained $K$ magnitudes by subtracting the typical $(R-K)$ colours of cluster ellipticals and spirals corrected for reddening (Smith et al. 2002). Finally, we converted to rest-frame luminosities by adopting $M_{K \odot}=3.28$ (Binney \& Merrifield 1998), a Galaxy extinction of $A_{K}=0.023$ (Schlegel et al. 1998), and using $K$-corrections from Mannucci et al. (2001).

To convert stellar luminosity into stellar mass we followed Lin et al. (2003): for ellipticals, we took the estimates of the central mass-to-light ratio as a function of galaxy luminosity from Gerhard et al. (2001); and for spiral galaxies, we used the values in Bell \& de Jong (2001). Estimating the mass-to-light ratios is the major source of uncertainty: different stellar population models predict stellar mass-to-light ratios as different as $0.7 M_{\odot} / L_{\odot}$ and $1.3 M_{\odot} / L_{\odot}$ (Cole et al. 2001). Additional errors are caused by either interlopers included in the catalogue or missed member galaxies. Furthermore, we did not consider stars contributing to the intracluster light, whose total fraction in AC 114 is $(11 \pm 2) \%$ in $r$ and $(14 \pm 3) \%$ in $B$ (Krick \& Bernstein 2007). It is then safe to consider an overall uncertainty $>40 \%$. The projected mass density in stars is plotted in Fig. 2. We performed the same kind of analysis described above for the number/luminosity density. Alike the luminosity density, the distribution of the mass 

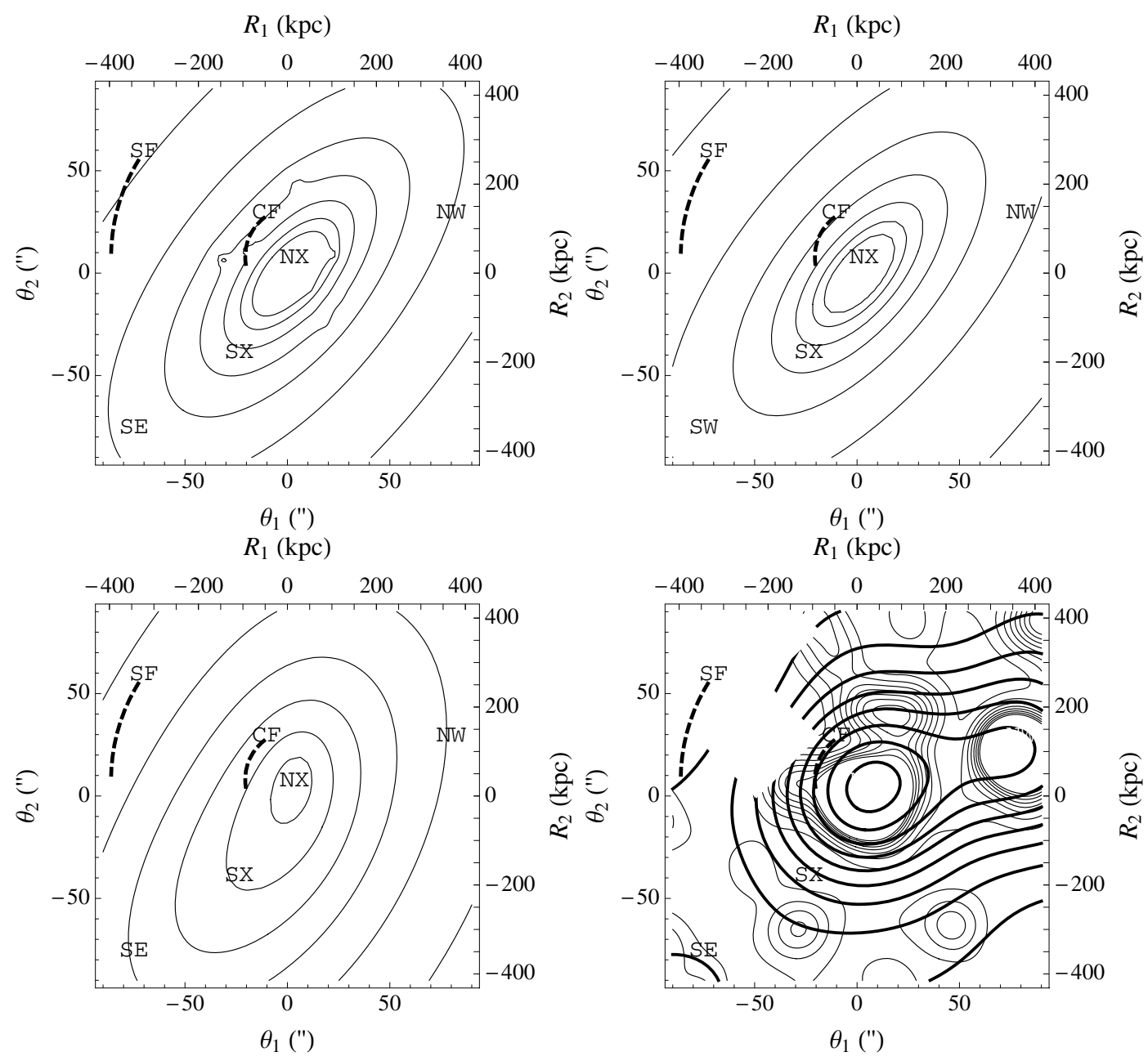

Fig. 2. Surface matter density distribution in the core region of AC 114, in units of the projected critical density for a source redshit at $z_{\mathrm{s}}=3.347$, $\Sigma_{\mathrm{cr}}=2154.2 M_{\odot} \mathrm{pc}^{-2}(h=0.7)$. Contours represent linearly spaced values of the convergence $\kappa$. NW and SE denote the positions of two galaxy clumps; NX and SX mark the location of the X-ray surface brightness peak for the main clump and the tail, respectively. The cold front CF and the shock front SF are plotted as dashed lines. Top-left: contour plot of the total matter density as inferred from lensing. $\kappa$-contours are plotted from 0.1 to 0.8 in steps of $\Delta \kappa=0.1$. Top-right: contours of the cluster-sized dark matter halo density as derived from the lensing analysis. $\kappa$-contour values go from 0.1 to 0.7 in steps of $\Delta \kappa=0.1$. Bottom-left: projected gas mass density derived from X-ray observations. The convergence contours run from 0.07 to 0.12 in steps of $\Delta \kappa=0.01$. Bottom-right: map of the projected mass density in stars derived from galaxy luminosities. The thick (thin) contours were obtained by smoothing the stellar mass density with a Gaussian kernel of dispersion $30^{\prime \prime}\left(10^{\prime \prime}\right) . k$-values run from $0.2 \times 10^{-2}$ to $1.8 \times 10^{-2}$ in steps of $0.2 \times 10^{-2}$ for the thick contours and from $2.5 \times 10^{-3}$ to $2.0 \times 10^{-2}$ in steps of $2 \times 10^{-3}$ for the thin contours.

in stars is elongated from northwest to southeast. The resulting integrated mass profile in the inner core is plotted in Fig. 5. The parameters of the distribution modelled as a King profile are reported in Table 1.

\section{Dynamics}

\subsection{Virial mass}

A dynamical estimate of the total mass can be derived using the virial theorem. Assuming the cluster to be approximately spherical, non-rotating, and in equilibrium, the virial mass can be expressed as (Binney \& Tremaine 1987)

$M_{\mathrm{V}}=\frac{3 \pi}{2} \frac{\sigma_{\mathrm{los}}^{2} R_{\mathrm{PV}}}{G}-C_{\mathrm{Pr}}$,

where $R_{\mathrm{PV}}$ is the projected virial radius of the observed sample of $N$ galaxies

$R_{\mathrm{PV}}=\frac{N(N-1)}{\sum_{i>j} R_{i j}^{-1}}$, and $R_{i j}$ is the projected distance between galaxies $i$ and $j$. The surface term $C_{\mathrm{Pr}}$ accounts for the system being not entirely enclosed in the observational sample. On average, for clusters observed to an aperture radius of $1.5 \mathrm{Mpc} / h$, the correction caused by $C_{\mathrm{Pr}}$ is $\sim 16 \%$ (Biviano et al. 2006).

Combining information about galaxy position and velocity, we derived estimates of $z_{\mathrm{cl}}=0.3153 \pm 0.0007$ for the cluster mean redshift and $\sigma_{\text {los }}=1900 \pm 100 \mathrm{~km} \mathrm{~s}^{-1}$ for the cluster velocity dispersion (see Appendix A). Non-members can strongly affect the mass estimate. Inclusion of interlopers that are currently infalling toward the cluster along filaments causes the overestimate of the harmonic mean radius, and, at the same time, the underestimate of the velocity dispersion. Using early-type galaxies as tracers might substantially reduce the interloper contamination in the virial mass estimate (Girardi \& Mezzetti 2001; Biviano et al. 2006). In our approach, we accounted for this issue by estimating the interloper fraction statistically. The error in $R_{\mathrm{PV}}$ was estimated by applying a statistical jackknife to the galaxy sample that passed the shifting gapper cut. The estimate of the cluster mass is then $M_{200}=(3.4 \pm 0.8) \times 10^{15} M_{\odot} / h$. 
An alternative mass estimator can be based entirely on the line-of-sight velocity dispersion. As inferred from fitting to simulated clusters, the $M_{200}-\sigma$ scaling relation is remarkably independent of cosmology. Using a cubic relation, Biviano et al. (2006) obtained

$M_{200}=(1.50 \pm 0.02)\left(\frac{\sqrt{3} \sigma_{\text {los }}}{10^{3} \mathrm{~km} \mathrm{~s}^{-1}}\right)^{3} \times 10^{14} h^{-1} M_{\odot}$

The intrinsic velocity distribution of early-type galaxies may be slightly biased relative to that of the dark matter particles (Biviano et al. 2006), so that when using Eq. (4) it is safer not to distinguish between morphological types. To properly apply the $M_{200}-\sigma$ relation in Eq. (4), we correct our estimate of the intrinsic velocity dispersion, which was obtained within a given observational aperture, according to the prescription of Biviano et al. (2006). We derive $M_{200}=(4.8 \pm 0.8 \pm 0.06) \times 10^{15} M_{\odot} / h$, where the second error is caused by the theoretical uncertainty in the relation. We will follow this convention throughout the paper. We can see how the two mass estimates of $M_{200}$ agree within the errors.

The concentration parameter can also be estimated using scaling relations fitted to numerical simulations as well. According to the scaling $c_{200} \sim 4\left[\sigma_{\text {los }} /\left(700 \mathrm{~km} \mathrm{~s}^{-1}\right)\right]^{-0.306}$ (Navarro et al. 1997; Biviano et al. 2006), where $\sigma_{\text {los }}$ is measured within an aperture radius of $1.5 \mathrm{Mpc} / h$, we find that $c_{200} \sim 2.95 \pm 0.05$.

\section{X-ray observations}

AC 114 has a strongly irregular X-ray morphology (De Filippis et al. 2004), see Fig. 1. The cluster does not exhibit a single $\mathrm{X}$-ray peak. Noticeable emission is associated with the cluster $\mathrm{cD}$ galaxy but the centroid of the overall X-ray emission is located about $10^{\prime \prime}$ northwest of the $\mathrm{cD}$ galaxy. Two principal components stand out: the cluster, roughly centred on the optical position, and a diffuse filament that spreads southeast for approximately $1.5^{\prime}(\sim 0.3 \mathrm{Mpc} / h)$, connecting the cluster core with the location of the SE clump (see Appendix B).

Additional signs of dynamical activity are observed northeast close to the cluster centre (see Fig. 2): a cold front at $\sim 20^{\prime \prime}$ $(\sim 70 \mathrm{kpc} / h)$ from the core centre and a likely shock front at $\sim 90^{\prime \prime}(\sim 0.3 \mathrm{Mpc} / h)$.

The tail and the fronts might be independent phenomena. Diffuse X-ray emission is detected near the SE clump, whereas no X-ray emission is associated with the NW clump. The NE substructure detected with the $\delta_{\mathrm{DS}}$-test (see Appendix B) was not targeted by X-ray observations. One possible scenario is that the SE clump, in its motion from the northwest through the cluster, has been ram-pressure stripped of most of its intra-group gas, now still visible as the soft southern tail. The interaction with the cluster might have also caused the asymmetrical stretch of the cluster emission detected toward the southeast. The NW clump might have been stripped as well.

The cluster bolometric luminosity is $L_{X}=\left(6.7_{-0.2}^{+0.4}\right) \times$ $10^{44} \mathrm{erg} \mathrm{s}^{-1} / h^{2}$ (De Filippis et al. 2004). Based on scaling relations between luminosity and velocity dispersion (Rykoff et al. 2008), we would expect $L_{X}=(5 \pm 1 \pm 1) \times 10^{45} \mathrm{erg} \mathrm{s}^{-1} / h^{2}$, which is higher than the observed value. This might suggest that on the one hand the gas has yet to settle in the cluster potential well and, on the other hand, the clumpy structure of AC 114 might lead to an overestimate of the velocity dispersion.

\subsection{Projected mass density}

The gas mass can be estimated from the X-ray emission. The surface brightness was modelled in De Filippis et al. (2004) as a sum of two elliptical isothermal $\beta$-profiles, a main core plus an extended off-centred south-east tail. To infer the projected mass associated with each component, we had to project the corresponding 3D ellipsoid, which were previously obtained by deprojecting the observed intensity map (see Appendix C). The resulting projected density profile for each component follows a King-like $\beta$ distribution (see Eq. (1)); parameters are listed in Table 2 . To obtain the corresponding three-dimensional electron density, we took care to deproject the surface brightness maps of the main clump and the tail separately. We then projected back the two separate distributions to the lens plane (see Appendix C for details). Since we treated the main clump and the tail separately, the only uncertainty in the projected mass caused by the casting method is then a correction geometrical factor depending on the unknown intrinsic axial ratios and orientation angles. The projected mass map is plotted in Fig. 2. The integrated ICM mass is plotted in Fig. 5.

The main sources of error in the gas mass are the projection effects and the assumption of isothermal emission. A lack of knowledge about the intrinsic axial ratios and the orientation of the gas distribution causes an uncertainty in the overall normalization of the projected gas density. In Appendix C, we estimate this uncertainty to be of the order of $\sim 18 \%$.

As expected from the several indications of dynamical activity, there is no evidence of a central cool core. The radial profile suggests a decline at large radii (De Filippis et al. 2004, see their figure 6), but because of the large errors a constant temperature is in full agreement with data. Furthermore, in the small central

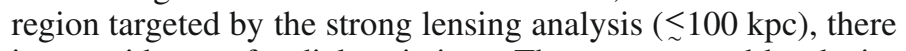
is no evidence of radial variations. The error caused by deviations from isothermality is a few percents and is much smaller than the uncertainty in the spectroscopic determination of the temperature.

In the inner core, the contribution of the tail is subdominant. We note that the central convergence for the ICM is $\kappa_{0} \lesssim 0.13$, so that the gas mass is subcritical for lensing.

\section{Strong lensing analysis}

\subsection{Optical data}

Many multiple image systems have been detected in the core of AC 114 (see Fig. 3). The first ones were discovered in a search for bright gravitational lensing arcs by Smail et al. (1991). Two images of the prominent three-image system $\mathrm{S}$ were first identified by Smail et al. (1995), whereas the third image S3 and the systems A, B, C, and D were discovered by Natarajan et al. (1998). The last image system E was located by Campusano et al. (2001), who also measured source redshifts using spectroscopic observations.

For our strong lensing model, we exploited only the image systems with confirmed spectroscopic redshift, i.e., A, E, and $\mathrm{S}$. The other systems were not considered, as they are strongly perturbed by some cluster galaxies or affected by a lack of precise redshift measurements. The image system $\mathrm{S}$, at redshift $z_{\mathrm{s}}=1.867$, is composed of three hook-shaped images (see Fig. 4). To take into account the parity and the orientation of the images and exploit the information carried by the shape, each $\mathrm{S}$-image was sampled by two points. We considered an uncertainty of $0.4^{\prime \prime}$, which will be the default error in each positional 
Table 2. Properties of the projected gas distribution as inferred from the X-ray analysis.

\begin{tabular}{lcrrrrrc}
\hline \hline Component & Mass scale & \multicolumn{1}{c}{$\begin{array}{c}\theta_{1,0} \\
\left({ }^{\prime \prime}\right)\end{array}$} & \multicolumn{1}{c}{$\begin{array}{c}\theta_{2,0} \\
\left({ }^{\prime \prime}\right)\end{array}$} & & $\begin{array}{c}\theta_{\epsilon} \\
(\mathrm{deg})\end{array}$ & $\begin{array}{c}\text { Length scale } \\
\left({ }^{\prime \prime}\right)\end{array}$ & $\beta$ \\
\hline Main Clump & $0.100 \pm 0.008$ & $3.7 \pm 1.0$ & $8.7 \pm 1.0$ & $0.39 \pm 0.05$ & $-13 \pm 4$ & $3.6 \pm 1.0$ & $0.389 \pm 0.007$ \\
Southern Tail & $0.033 \pm 0.006$ & $-23.4 \pm 1.0$ & $-37.6 \pm 1.0$ & $0.50 \pm 0.02$ & $-37 \pm 2$ & $170 \pm 40$ & $1.8 \pm 1.0$ \\
\hline
\end{tabular}

Notes. Each component was modelled as an isothermal $\beta$-ellipsoid.

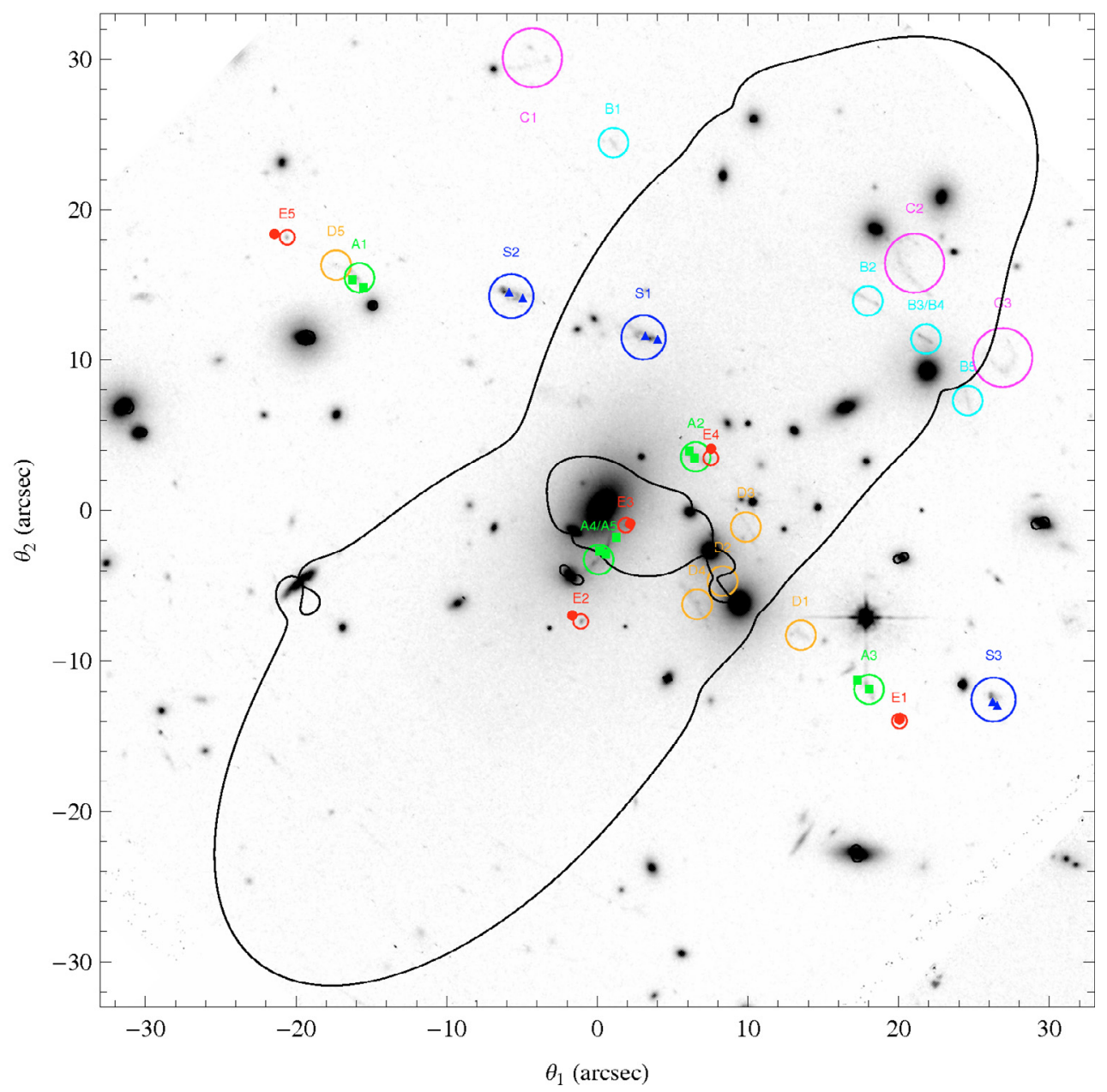

Fig. 3. HST/WFPC-2 image of AC 114 with the observed multiple image systems. The coordinates $\theta_{1}$ and $\theta_{2}$, both measured in arcseconds are in directions west and north, respectively. The critical lines are represented by the black lines and are referred to the image system $\mathrm{E}\left(z_{\mathrm{s}}=3.347\right)$. Circles surround multiple images (A, B, C, D, E, and S systems), while the filled squares, small circles, and triangles mark the predicted image positions for the system A (green in the electronic version of the paper), E (red), and S (blue), respectively.

data point. The image system $\mathrm{E}$ is composed of five nearly pointlike images at redshift $z_{\mathrm{s}}=3.347$, see Fig. 4. The multiple image system A consists of five images of a single source at redshift $z_{\mathrm{s}}=1.691$. The images A1, A2, and A3 are only weakly stretched by the lens, and it can be seen that morphologically they are images of the same source. We distinguished two conjugate knots in each image, see Fig. 4. On the other hand, A4 and A5 are strongly stretched because they merge into a single arc across the radial critical curve near the BCG. As the knots in these two central images cannot be distinguished, they have been furnished with a larger uncertainty $\left(1.6^{\prime \prime}\right)$.

The adopted positional uncertainties are larger than the HST astrometric resolution. Clusters are complex systems and simple models cannot account for all the mass complexities. A coarser positional error allows us to perform the lensing analysis without

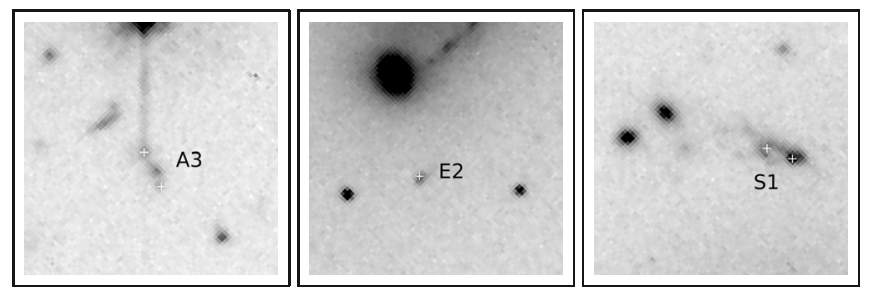

Fig. 4. A mosaic of the zoomed-in regions $\left(\sim 8^{\prime \prime} \times 8^{\prime \prime}\right)$ surroundings the images A3 (left panel), E2 (middle panel), and S1 (right panel). A3 has an elongated shape wherein distinct points can be recognized. For E2, the intrinsic morphology of the source cannot be distinguished, whereas system S images are hook-shaped, as shown here for S1. The white crosses are the coordinates of the sampled points used into the strong lensing analysis. 
adding too many parameters and, at the same time, avoiding the explored region in parameter space being overly confined (Sand et al. 2008). This approach can be effective when dealing with galaxy clumps such as those found in the AC 114 luminosity map, which are usually associated with meso-structures (Saha et al. 2007).

\subsection{Mass components}

We performed a strong lensing analysis exploiting the available optical observations (see Sect. 2), and measurements in the X-ray band (see Sect. 4). This multiwavelength approach allowed us to model the three main components: the cluster-sized dark matter halo, the cluster-sized ICM, and the observed galaxies. Each component was described with a separate parametric mass model.

The projected surface mass density $\Sigma$ of these density profiles is expressed in terms of the convergence $\kappa$, i.e., in units of the critical surface mass density for lensing, $\Sigma_{\mathrm{cr}}=$ $\left(c^{2} D_{\mathrm{s}}\right) /\left(4 \pi G D_{\mathrm{d}} D_{\mathrm{ds}}\right)$, where $D_{\mathrm{s}}, D_{\mathrm{d}}$, and $D_{\mathrm{ds}}$ are the source, the lens, and the lens-source angular diameter distances, respectively. We considered mass distributions with elliptical symmetry, so that the convergence can be written in terms of the elliptical radius $\theta_{\text {ell }}$.

To model the cluster-sized DM component, we considered parametric mass models with either isothermal or NavarroFrenk-White (NFW) density profiles. DM halos can be successfully described as NFW profiles (Navarro et al. 1996, 1997), whose 3D distribution follows

$\rho_{\mathrm{NFW}}=\frac{\rho_{\mathrm{s}}}{\left(r / r_{\mathrm{s}}\right)\left(1+r / r_{\mathrm{s}}\right)^{2}}$,

where $\rho_{\mathrm{s}}$ is the characteristic density and $r_{\mathrm{s}}$ is the characteristic length scale. The convergence of this mass profile with elliptical symmetry is obtained by replacing the polar with the elliptical radius in the resulting projected surface mass density. We described the projected NFW density in terms of the strength of the lens $\kappa_{\text {NFW }}$ (see Eq. (C.7)), and of the projected length scale $r_{\mathrm{sP}}$ (see Appendix C), i.e., the two parameters directly inferred by fitting projected lensing maps.

An alternative description of a DM component is given in terms of isothermal mass density. The non-singular isothermal profiles are parametrized by a softened power-law ellipsoid (NIE), and represent a special case of $\beta$-models with $\beta=2 / 3$ (see Eq. (1)). The mass scale parameter is usually written as $b=2 \kappa_{0} r_{\mathrm{cP}}$ (Keeton 2001b), where $\kappa_{0}$ is the central convergence and $r_{\mathrm{cP}}$ is the projected core radius.

The two gas components, i.e., the main X-ray clump and the soft tail, can be modelled as $\beta$-profiles, see Eq. (1). Unlike the DM component, which was modelled as isothermal, the slope for each gas component is fixed by the X-ray observations, see Table 2. We note that the mass distribution of the main X-ray emitting clump is quite flat, so that the impact on lensing features is limited. We considered a normal prior on the mass normalization $\kappa_{0}$ of the main gas component whose mean and dispersion had been determined from the X-ray analysis, see Table 2, and sharp priors on the remaining parameters describing the ICM distribution.

To perform an accurate lens modelling, we also have to consider the mass distribution of the galaxies. Galaxies are small compared to the whole cluster, but have high local mass densities and can strongly perturb the cluster potential in their neighborhood. Galaxies affecting the considered image systems must therefore be taken into account. Our galaxy selection was limited to the region of the cluster where the multiple image systems are located. We selected galaxies brighter than $R_{702}=21.2$ within a radius of $44^{\prime \prime}$ from the BCG finding that 25 galaxies passed the cut.

Galaxy-sized halos can be modelled by pseudo-Jaffe mass profiles, which are obtained by subtracting a NIE of core radius $r_{\mathrm{t}}$ (called truncation radius) from another NIE of core radius $r_{\mathrm{c}}$, where $r_{\mathrm{c}}<r_{\mathrm{t}}$. Apart from the BCG, see Sect. 2, we considered spherical galaxies. Each pseudo-Jaffe model was characterised by a velocity dispersion $\sigma_{\mathrm{DM}}$, a core radius $r_{\mathrm{c}}$ and a truncation radius $r_{\mathrm{t}}$. To minimise the number of parameters, a set of scaling laws was adopted (Brainerd et al. 1996): $\sigma_{\mathrm{DM}}=\sigma_{\mathrm{DM}}^{*}\left(L / L^{*}\right)^{1 / 4}$ and $r_{\mathrm{t}}=r_{\mathrm{t}}^{*}\left(L / L^{*}\right)^{1 / 2}$. The core radius $r_{\mathrm{c}}$ was scaled in the same way as $r_{\mathrm{t}}$. The dispersion $\sigma_{\mathrm{DM}}$ was related to the total mass using $M=(9 / 2 G) \sigma_{\mathrm{DM}}^{2} r_{\mathrm{t}}$ (Natarajan et al. 1998). To define a characteristic luminosity $L^{*}$, we considered a hypothetical galaxy with $R_{702}=19.5$. We considered a flat prior on $\sigma_{\mathrm{DM}}^{*}$, which was left to vary between 0 and $450 \mathrm{~km} \mathrm{~s}^{-1}$, whereas $r_{\mathrm{c}}^{*}$ and $r_{\mathrm{t}}^{*}$ were fixed to $0.15 \mathrm{kpc}$ and $45 \mathrm{kpc}$, respectively (Natarajan et al. 2009).

Being a distinct galaxy population from $L^{*}$ cluster ellipticals, BCGs should be modelled on their own. Treating the BCGs as average cluster members may introduce additional uncertainty when studying the mass-to-light ratios of typical earlytype galaxies (Natarajan et al. 1998). However, as far as ellipticity, orientation, and centroid (the main features we are going to compare among the different mass components) of the clustersized DM halo are concerned, a different lensing modelling of the BCG would have no significant impact.

In general, modelling each perturbing galaxy on its own would allow us to obtain a closer fit to the data (Limousin et al. 2008). On the other hand, the cluster-sized DM halo cannot be effectively distinguished from that of the BCG by means of pure lensing analyses ${ }^{1}$. As far as a regular cluster is concerned, one could assume that the two halos are centred at the same position and then model only the stellar content of the BCG in the lensing model. In this way, the cluster-sized DM halo would also account for the BCG dark halo.

We explored the mass components in AC 114 without forcing the DM distribution to follow either the gas or the galaxy density and we allowed the DM centroid to be free to vary. Since a reasonable physical model requires DM to be associated with the BCG, we had to account also for the BCG halo. However, in the present paper, we were mainly concerned with the clustersized DM component so that we preferred to keep the number of free parameters linked to galactic halos as small as possible. We then considered three different modellings. As a first case, the BCG was modelled on its own as a pseudo-Jaffe profile with ellipticity fixed by his luminosity distribution and velocity dispersion modelled after imposing a flat prior $0<\sigma_{\mathrm{DM}} \leq 500 \mathrm{~km} \mathrm{~s}^{-1}$. Alternatively, we constrained the BCG total mass to follow the same scaling relations as the other galaxies. With this scaling, the NFW cluster-sized profile represented most of the DM associated with the BCG halo. In both cases, the core and the truncation radius were scaled according to their characteristic values. This has a negligible effect because of the degeneracy between the scale-length and the velocity dispersion. As a final case, we assumed that the total BCG mass distribution is embedded in the cluster-sized dark matter halo. We note that this worked only for the cusped NFW halo.

${ }^{1}$ Dynamical analyses of the inner velocity profile are required to differentiate the cluster-sized from the BCG contribution (Sand et al. 2008). 
Our analysis is not meant to investigate whether the BCG can be included as either part of the main cluster or a separate potential. The above-mentioned tested models differ in the values of the central velocity dispersion profile and should be distinguished by exploiting dynamical analyses in the very inner regions. We considered these very different cases of BCG modelling to demonstrate that the impact of gas in lens modelling is nearly independent of the galaxies. However, we emphasise that the gas and stellar mass distribution, discussed in Sect. 7, were inferred with tools independent of the lensing analysis.

\section{Inferred mass distribution}

To accomplish the strong lensing analysis, we performed a Bayesian investigation. The parameter probability distributions were then determined studying the posterior function. Computation of the likelihood function was based on the gravlens software (Keeton 2001a,b). This analysis was performed in the source plane. Because of the large number of parameters and models, we exploited the Laplace approximation (Mackay 2003). The total number of constraints (42) equalled the number of coordinates of the observed image positions. The number of free parameters, which was allowed to vary, i.e., the free parameters in the mass models plus the (10) unknown coordinates of the source positions, is 16 for a lens model with just a single cluster-sized halo. An additional parameter may account for the velocity dispersion of the scaled galactic halos. As far as the BCG is concerned, we do not add parameters if the BCG is embedded in the cluster-sized halo or forced to follow the scaling law; otherwise, we add one additional parameter if the velocity dispersion is allowed to vary. Finally, some other parameters account for the gas distribution when the ICM is modelled on its own. As a priori distribution for the parameters of the ICM, we consider the results from the X-ray analysis.

In the present first attempt to include the ICM in a lensing analysis, we considered how and if the inclusion of gas improves the lensing modelling. An efficient way to compare different models is to consider the Bayesian evidence $E$ (Mackay 2003). A difference of 2 for $\log E$ is regarded as positive evidence, and of 6 or more as strong evidence, compared to the model with the smaller value.

We note that performing the fitting to just a single image system leads to a very small $\chi^{2}$-value for all the considered models independently of the system (A, E, or S), since constraints associated with a single system are not enough to reliably determine the parameters. Only analysing all the image systems simultaneously provides a reliable constraint of the mass models.

\subsection{NFW profile}

We first considered NFW profiles. Models consisted of a clustersized NFW distribution and additional components for the galactic halos and the ICM, see Table 3. Notation in Table 3 and in the following discussion distinguishes models according to the matter components included in the cluster-sized halo and the modelling of the BCG. The NFW main halo describes the diluted DM plus possible additional contributions. It can encompass either: i) all the components at the same time (in the "all" model, DM and baryons are described by only one NFW profile); ii) just the DM ("halo"); iii) DM and gas ("halo with ICM"); iv) DM and gas and BCG ("halo with ICM and BCG"); or, finally, v) diluted DM plus all galactic halos ("halo with all galaxies") or vi) plus only the BCG ("halo with BCG"). When modelled apart, the galaxies can account for the BCG plus other ellipticals fitting a single scaling law ("all galaxies") or just the other ellipticals without the BCG ("other galaxies").

The simplest model is a single NFW halo, representing the total matter distribution (DM+ICM+galaxies). Its parameters are listed in Table 3 (see the "all" model). Even if the value of the scale length is larger than the range over which observational constraints are found, a combined fit to multiple source redshift image systems allows us to determine $r_{\mathrm{sP}}$ and its uncertainty (Limousin et al. 2008). This is an important step in estimating the concentration (see Sect. 8.2). With this simple mass model, we were able to reproduce the observed images far more successfully than assuming a single isothermal profile (see Sect. 6.2). All the images were reproduced with a mean distance of $\simeq 0.6^{\prime \prime}$. Since all the priors on the parameters are flat, it makes sense to consider the $\chi^{2}(\simeq 32.1)$ of the inferred model. Even for a quite complex system such as AC 114 a single NFW model, accounting at the same time for dark matter, stars, and gas, can provide a good fit to the data with a reduced $\chi_{\text {red }}^{2} \simeq 1.2$.

The subsequent addition of ICM and galaxy-sized halos considerably improved the fit, but above all helped to achieve a physically more consistent model, which describes the features of the cluster more accurately. As a first step, we followed the usual approach and considered galactic halos together with a cluster-sized component. In these models ("halo with ICM" and "halo with ICM and BCG" in Table 3), all the diluted mass distributions, i.e., dark matter plus gas, contribute to a single clustersized NFW halo. The fitted parameters for each component are listed in Table 3. By allowing the velocity dispersion of the BCG to vary, the degeneracy between the cusped cluster-sized halo and the BCG halo takes over and the posterior probability is maximum for a $\mathrm{cD}$ galaxy with null mass. We then limited our analysis to a BCG halo either following galactic scaling laws ("halo with ICM" and "all galaxies") or embedded in the clustersized one ("halo with ICM and BCG" and "other galaxies"). In both cases, the evidence factor is larger than that for the case a single NFW halo ("all" model). We note that the listed values of the evidence are given apart from a constant factor depending on the data and a second hidden factor depending on the flat priors on the parameters of the NFW profile, which is constant across the models.

As a second step, we considered the effect of explicitly modelling the gas distribution. For all the analysed models, by adding a component for the ICM the evidence becomes larger. In Bayesian analysis, given equal priors for the different hypotheses, model are ranked by evaluating the evidence. Then, from a statistical point of view it is better to model the gas independently of the DM cluster-sized halo. The physical reason beyond that is that the ICM does not follow the mass. In a relaxed cluster, the gas follows the gravitational potential and is rounder than the mass distribution. Our analysis demonstrates that the differences between gas and dark matter distribution are further exacerbated if the cluster is dynamically active.

Models whose cluster-sized halo has to account for: i) either DM+gas+galaxies ("all"); or ii) DM+BCG+gas with other galaxies modelled separately ("halo with ICM and BCG" and "other galaxies"), or iii) DM+galaxies with gas modelled separately ("halo with all galaxies" with "ICM"); or iv) DM+BCG with other galaxies and gas modelled separately ("halo with BCG" and "other galaxies" and "ICM") have an evidence factor of $\log E \sim-12.4,-7.6$, and -4.0 or 0.0 , respectively. Adding physically motivated components increases the evidence. This trend is also confirmed by a different version of the model where the cluster-sized components account only for the diluted DM, whereas the BCG follows the galactic scaling laws and the ICM 
Table 3. Model parameters inferred from the lensing analysis.

\begin{tabular}{|c|c|c|c|c|c|c|c|c|}
\hline Component & Mass scale & $\begin{array}{l}\theta_{1,0} \\
\left({ }^{\prime \prime}\right)\end{array}$ & $\begin{array}{l}\theta_{2,0} \\
\left({ }^{\prime \prime}\right)\end{array}$ & $\epsilon$ & $\begin{array}{c}\theta_{\epsilon} \\
(\mathrm{deg})\end{array}$ & $\begin{array}{c}\text { length scale } \\
\text { (") }\end{array}$ & & $\log E$ \\
\hline All & $\kappa_{\mathrm{NFW}}$ & & & & & $r_{\mathrm{sP}}$ & & \\
\hline NFW & $0.213 \pm 0.017$ & $1.50 \pm 0.17$ & $-0.5 \pm 0.2$ & $0.50 \pm 0.03$ & $-38.3 \pm 0.4$ & $210 \pm 30$ & - & -12.4 \\
\hline Halo with ICM & $\kappa_{\mathrm{NFW}}$ & & & & & $r_{\mathrm{sP}}$ & & \\
\hline NFW & $0.195 \pm 0.017$ & $1.0 \pm 0.3$ & $-0.6 \pm 0.3$ & $0.54 \pm 0.03$ & $-37.2 \pm 0.6$ & $240 \pm 40$ & - & -9.2 \\
\hline All galaxies & $\sigma_{\mathrm{DM}}\left(\mathrm{km} \mathrm{s}^{-1}\right)$ & & & & & $r_{\mathrm{cP}}(\mathrm{kpc})$ & $r_{\mathrm{tP}}(\mathrm{kpc})$ & \\
\hline pJaffe- $L^{*}$ & $85 \pm 19$ & - & - & - & - & {$[0.15]$} & [45] & \\
\hline Halo with ICM and BCG & $\kappa_{\mathrm{NFW}}$ & & & & & $r_{\mathrm{sP}}$ & & \\
\hline NFW & $0.205 \pm 0.016$ & $1.1 \pm 0.3$ & $-0.3 \pm 0.3$ & $0.52 \pm 0.03$ & $-37.1 \pm 0.7$ & $220 \pm 30$ & - & -7.6 \\
\hline Other galaxies & $\sigma_{\mathrm{DM}}\left(\mathrm{km} \mathrm{s}^{-1}\right)$ & & & & & $r_{\mathrm{cP}}(\mathrm{kpc})$ & $r_{\mathrm{tP}}(\mathrm{kpc})$ & \\
\hline pJaffe- $L^{*}$ & $90 \pm 20$ & - & - & - & - & {$[0.15]$} & [45] & \\
\hline $\begin{array}{l}\text { Halo with all galaxies } \\
\text { NFW }\end{array}$ & $\begin{array}{c}\kappa_{\mathrm{NFW}} \\
0.206 \pm 0.016\end{array}$ & $1.47 \pm 0.17$ & $-0.48 \pm 0.19$ & $0.51 \pm 0.03$ & $-40.6 \pm 0.6$ & $\begin{array}{c}r_{\mathrm{sP}} \\
170 \pm 20\end{array}$ & - & -4.0 \\
\hline ICM & $\kappa_{0}$ & & & & & $r_{\mathrm{cP}}$ & $\beta$ & \\
\hline Main Clump & $0.094 \pm 0.011$ & {$[3.7]$} & [8.7] & {$[0.39]$} & {$[-13]$} & {$[13.6]$} & [0.389] & \\
\hline Southern Tail & {$[0.033]$} & {$[-23.4]$} & {$[-37.6]$} & {$[0.50]$} & {$[-37]$} & [170] & [1.8] & \\
\hline Halo & $\kappa_{\mathrm{NFW}}$ & & & & & $r_{\mathrm{sP}}$ & & \\
\hline NFW & $0.190 \pm 0.019$ & $1.2 \pm 0.5$ & $-0.4 \pm 0.3$ & $0.53 \pm 0.03$ & $-40 \pm 1$ & $190 \pm 30$ & - & -1.9 \\
\hline All galaxies & $\sigma_{\mathrm{DM}}\left(\mathrm{km} \mathrm{s}^{-1}\right)$ & & & & & $r_{\mathrm{cP}}(\mathrm{kpc})$ & $r_{\mathrm{tP}}(\mathrm{kpc})$ & \\
\hline pJaffe- $L^{*}$ & $70 \pm 30$ & - & - & - & - & {$[0.15]$} & [45] & \\
\hline ICM & $\kappa_{0}$ & & & & & $r_{\mathrm{cP}}$ & $\beta$ & \\
\hline Main Clump & $0.093 \pm 0.013$ & [3.7] & [8.7] & [0.39] & {$[-13]$} & [13.6] & [0.389] & \\
\hline Southern Tail & [0.033] & {$[-23.4]$} & {$[-37.6]$} & {$[0.50]$} & {$[-37]$} & {$[170]$} & {$[1.8]$} & \\
\hline Halo with BCG & $\kappa_{\mathrm{NFW}}$ & & & & & $r_{\mathrm{sP}}$ & & \\
\hline NFW & $0.198 \pm 0.017$ & $1.2 \pm 0.3$ & $-0.3 \pm 0.3$ & $0.53 \pm 0.03$ & $-39.4 \pm 0.9$ & $180 \pm 30$ & - & 0.0 \\
\hline Other galaxies & $\sigma_{\mathrm{DM}}\left(\mathrm{km} \mathrm{s}^{-1}\right)$ & & & & & $r_{\mathrm{cP}}(\mathrm{kpc})$ & $r_{\mathrm{tP}}(\mathrm{kpc})$ & \\
\hline pJaffe & $80 \pm 20$ & - & - & - & - & {$[0.15]$} & [45] & \\
\hline ICM & $\kappa_{0}$ & & & & & $r_{\mathrm{cP}}$ & $\beta$ & \\
\hline Main Clump & $0.092 \pm 0.013$ & [3.7] & [8.7] & [0.39] & {$[-13]$} & [13.6] & {$[1.833]$} & \\
\hline Southern Tail & [0.033] & {$[-23.4]$} & {$[-37.6]$} & {$[0.50]$} & {$[-37]$} & {$[170]$} & {$[-2.4]$} & \\
\hline
\end{tabular}

Notes. For each model, the first line specifies the mass components accounted by the cluster-sized halo, parameterized as a NFW distribution."All" refers to a mass model (DM+baryons) with a single NFW component. The other models may have additional components either for the gas or the galactic halos. The BCG is either scaled as the other galaxies ("All galaxies") or embedded in the cluster-zised halo (halos "with BCG"). The ICM is either modelled apart or embedded in the cluster-sized halo ("Halo with ICM"). The orientation angle $\theta_{\epsilon}$ is measured north over east and $r_{\mathrm{SP}}\left(r_{\mathrm{cP}}\right)$ is the projected length scale (core radius) for the NFW $(\beta)$ profile. Parameters in squared brackets were kept fixed in the lensing analysis. Convergences are normalised to the E system source redshift.

is modelled separately ("halo" and "galaxies with BCG" and "ICM"). The corresponding evidence ( $\log E \sim-1.9)$ is larger than those of models without either the galaxies or the gas. This confirms that the results are independent of the modelling of the BCG.

Since accounting for the gas is quite unusual in lensing analyses, we compare the models accounting for a gas component in the usual way, i.e., a composite mass distribution in which both ICM and DM are parameterized altogether as a single NFW profile. This conventional model provides a close fit to the data either for the BCG scaled together with the other galaxies ("Halo with ICM" and "All galaxies", $\chi^{2} \simeq 24.8$ ) or for the BCG embedded in the cluster-sized halo ("Halo with ICM and BCG" and "Other galaxies", $\chi^{2} \simeq 21.8$ ). We note that the inclusion of both galactic and ICM components is needed to improve the fit, whereas accounting only for the gas is not helpful. It is the physical information obtained from X-ray data that infers we need to include the ICM in the modelling. Our analysis shows that adding physically motivated complexity to the lensing models (either in the form of galactic halos or in the form of diluted gas distribution) improves the description of a cluster lens from both the statistical and the physical point of view.

We note that as far as a simple $\chi^{2}$-analysis goes, adding the gas component would not be justified for AC 114, since the fit is not significantly improved. This point needs to be investigated further by considering a sample of clusters with different X-ray surface brightness slopes.

Modelling the gas helps us to more clearly investigate the DM halo. Comparing the properties of the cluster-sized DM halos (with or without galaxies), see Table 3, to DM+gas halos (with or without galaxies), two properties stand out. First, to account for the mass contributed by the gas, the DM+ICM halo has a larger central convergence and larger radius. The two parameters vary accordingly in such a way as to leave the 
Table 4. Model parameters inferred from the lensing analysis when the cluster-sized halo is modelled as a NIE and $r_{\mathrm{cP}}$ is the projected core radius.

\begin{tabular}{|c|c|c|c|c|c|c|c|c|}
\hline Component & mass scale & $\begin{array}{l}\theta_{1,0} \\
\left({ }^{\prime \prime}\right)\end{array}$ & $\begin{array}{l}\theta_{2,0} \\
\left({ }^{\prime \prime}\right)\end{array}$ & $\epsilon$ & $\begin{array}{c}\theta_{\epsilon} \\
(\mathrm{deg})\end{array}$ & $\begin{array}{c}\text { length scale } \\
\text { (") }\end{array}$ & & $\log E$ \\
\hline Halo with ICM & $b\left({ }^{\prime \prime}\right)$ & & & & & $r_{\mathrm{sP}}$ & & -13.7 \\
\hline NIE & $27 \pm 2$ & $0.2 \pm 0.7$ & $-0.4 \pm 0.7$ & $0.59 \pm 0.04$ & $-35.2 \pm 0.6$ & $11.9 \pm 1.4$ & - & \\
\hline Galaxies & $\sigma_{\mathrm{DM}}\left(\mathrm{km} \mathrm{s}^{-1}\right)$ & & & & & $r_{\mathrm{cP}}(\mathrm{kpc})$ & $r_{\mathrm{tP}}(\mathrm{kpc})$ & \\
\hline pJaffe- $L^{*}$ & $147 \pm 13$ & - & - & - & - & [0.15] & [45] & \\
\hline Halo with ICM & $b\left(^{(\prime)}\right)$ & & & & & $r_{\mathrm{sP}}$ & & -6.7 \\
\hline NIE & $31 \pm 3$ & $0.8 \pm 0.8$ & $-0.6 \pm 0.7$ & $0.55 \pm 0.04$ & $-36.4 \pm 0.6$ & $13.7 \pm 1.4$ & - & \\
\hline Galaxies & $\sigma_{\mathrm{DM}}\left(\mathrm{km} \mathrm{s}^{-1}\right)$ & & & & & $r_{\mathrm{cP}}(\mathrm{kpc})$ & $r_{\mathrm{tP}}(\mathrm{kpc})$ & \\
\hline $\begin{array}{l}\text { pJaffe- } L^{*} \\
\text { pJaffe-BCG }\end{array}$ & $\begin{array}{l}110 \pm 20 \\
239 \pm 18\end{array}$ & $\begin{array}{c}- \\
{[0.0]}\end{array}$ & $\stackrel{-}{[0.0]}$ & $\overline{-}$ & {$[-\overline{32.8}]$} & $\begin{array}{l}{[0.15]} \\
{[0.29]}\end{array}$ & $\begin{array}{l}{[45]} \\
{[87]}\end{array}$ & \\
\hline Halo & $b\left(^{\prime \prime}\right)$ & & & & & $r_{\mathrm{sP}}$ & & -5.9 \\
\hline NIE & $23.3 \pm 1.3$ & $0.4 \pm 0.6$ & $-0.1 \pm 0.6$ & $0.57 \pm 0.04$ & $-37.3 \pm 0.8$ & $11.1 \pm 1.2$ & - & \\
\hline Galaxies & $\sigma_{\mathrm{DM}}\left(\mathrm{km} \mathrm{s}^{-1}\right)$ & & & & & $r_{\mathrm{cP}}(\mathrm{kpc})$ & $r_{\mathrm{tP}}(\mathrm{kpc})$ & \\
\hline pJaffe- $L^{*}$ & $137 \pm 12$ & - & - & - & - & {$[0.15]$} & [45] & \\
\hline ICM & $\kappa_{0}$ & & & & & $r_{\mathrm{cP}}$ & $\beta$ & \\
\hline Main Clump & $0.091 \pm 0.015$ & [3.7] & [8.7] & {$[0.39]$} & {$[-13]$} & [13.6] & [0.389] & \\
\hline Southern Tail & [0.033] & {$[-23.4]$} & {$[-37.6]$} & {$[0.50]$} & {$[-37]$} & {$[170]$} & {$[1.8]$} & \\
\hline Halo & $b\left(^{\prime \prime}\right)$ & & & & & $r_{\mathrm{SP}}$ & & 3.2 \\
\hline NIE & $26 \pm 2$ & $1.1 \pm 0.8$ & $-0.3 \pm 0.7$ & $0.53 \pm 0.04$ & $-39.0 \pm 1.1$ & $12.9 \pm 1.0$ & - & \\
\hline Galaxies & $\sigma_{\mathrm{DM}}\left(\mathrm{km} \mathrm{s}^{-1}\right)$ & & & & & $r_{\mathrm{cP}}(\mathrm{kpc})$ & $r_{\mathrm{tP}}(\mathrm{kpc})$ & \\
\hline $\begin{array}{l}\text { pJaffe- } L^{*} \\
\text { pJaffe-BCG }\end{array}$ & $\begin{array}{l}140 \pm 40 \\
227 \pm 18\end{array}$ & $\overline{[0.0]}$ & $\overline{[0.0]}$ & {$[0.42]$} & {$[-\overline{-} \cdot 8]$} & $\begin{array}{l}{[0.15]} \\
{[0.29]}\end{array}$ & $\begin{array}{l}{[45]} \\
{[87]}\end{array}$ & \\
\hline ICM & $\kappa_{0}$ & & & & & $r_{\mathrm{cP}}$ & $\beta$ & \\
\hline Main Clump & $0.093 \pm 0.012$ & [3.7] & [8.7] & [0.39] & {$[-13]$} & [13.6] & [0.389] & \\
\hline Southern Tail & [0.033] & {$[-23.4]$} & {$[-37.6]$} & {$[0.50]$} & {$[-37]$} & [170] & {$[1.8]$} & \\
\hline
\end{tabular}

Notes. The BCG is scaled either in the same way as the other galaxies or varied independently (see models with a "pJaffe-BCG" component). The ICM is either modelled apart ("Halo") or embedded in the cluster-sized halo ("Halo with ICM").

concentration nearly unchanged. Second, because of the misalignment between gas and DM, the DM+gas halo turns out to be rotated counter-clockwise with respect to the only DM component.

The addition of the ICM, which is quite flattened, caused a slight decrease in the projected scale length, $r_{\mathrm{sP}}$, of the DM component, whose orientation experienced a clockwise rotation of $\sim 2 \mathrm{deg}$. These changes are small, as the ICM has a relatively low mass compared to the dark matter, but nevertheless interesting. The decrease in $r_{\mathrm{sP}}$ shows that the dark matter component is more compact than the ICM. The total projected mass within $75 \mathrm{kpc}(150 \mathrm{kpc})$ is $[3.8 \pm 0.4] \times 10^{13} M_{\odot}\left([11.3 \pm 1.0] \times 10^{13} M_{\odot}\right)$, in good agreement with previous estimates (Natarajan et al. 1998; De Filippis et al. 2004).

The ellipticity and orientation of the dark matter component are almost the same as those of the southern component of the ICM, whereas the northern component, which is the main baryonic component in the cluster core, is less elliptical and rotated counter-clockwise by $\sim 27 \mathrm{deg}$ compared to the dark matter. Its centroid is displaced $\sim 40 \mathrm{kpc}$ from the centre of the dark matter distribution. This clearly evident spatial offset between the dark matter and the main baryonic component in the cluster core provides evidence that the cluster is not in equilibrium. That between the centre of the dark matter component and the position of the BCG there is no significant offset portends that the dark matter behaves like collisionless particles during the merging process.
We can test the predictive power of our model by guessing the source redshifts of the multiple image systems without spectroscopic confirmation. Both the B and D systems are strongly perturbed by local galaxies and a prediction would require a detailed modelling of galactic halos, which is beyond the scope of our analysis. The system $\mathrm{C}$ is not affected by this problem. This three image system has a predicted lensing redshift of $\sim 2.3$ in agreement with Campusano et al. (2001).

\subsection{Isothermal profile}

Alternatively to the NFW model, we considered an isothermal profile for the main mass component (see Table 4). When the BCG is modelled apart from the other galaxies an additional entry line shows up ("pJaffe-BCG"); in the absence of this line, the BCG halo follows the standard scaling law for ellipticals. As a first step, we modelled AC 114 with a single NIE, representing all the matter present in the galaxy cluster. As in the case of NFW parameters, we used flat priors. This model, which turned out to be centred on the neighbourhood of the BCG galaxy, was quite inadequate. The reason is that the central density of this model is too low and therefore the central caustic too narrow, which in turn causes the merging images A4 and A5 to vanquish.

To solve this issue we added the mass distribution from galaxy-sized halos. In contrast to the cusped NFW, a cored NIE needs an additional peaked mass distribution associated with the 
BCG to provide a good fit to the data. Owing to the degeneracy between BCG and cluster-sized DM halo, it is then misleading to interpret the DM distributions studied in this section as pure cored isothermal ones. Because of the the small core radius imposed on the BCG, the overall profile has an effective central divergence that comes afloat from the cored NIE. The distribution is isothermal, $\rho \simeq r^{-2}$, only at large radii $\left(\gtrsim 12^{\prime \prime}\right)$. We note that the fit, and consequently the evidence, improves significantly when the BCG is not constrained to follow the galactic scaling laws.

We finally considered separately the ICM, the galaxy sized halos, and the dark matter component that was modelled as a NIE profile. The total projected mass within the circle of radius $75 \mathrm{kpc}(150 \mathrm{kpc})$ is $[4.0 \pm 0.3] \times 10^{13} M_{\odot}\left([11.2 \pm 0.9] \times 10^{13} M_{\odot}\right)$, which agrees with the estimate based on the assumption of DM distributed as a NFW profile and with previous results in Campusano et al. (2001), who used a slightly different modelling, i.e., pseudo-Jaffe profiles for both galactic and clustersized halos. We note that they had to consider additional NW and SE substructures to account for weak lensing effects outside of the very inner core.

As in the NFW case, the addition of the ICM mass components did not significantly improve the $\chi^{2}$, since its mass distribution is widely distributed, with a subcritical surface density that is unable to produce any strong lensing. Only its total mass has an influence on the lensing properties of the cluster. On the other hand, the evidence factor increases for each subsequent addition of physically motivated components. Models with an explicit component for the ICM have larger evidences than corresponding models without. The result is then independent of the parameterization of the DM halo.

The value of $\sigma_{\mathrm{DM}}$ for the $L^{*}$ galaxy has to be much higher when assuming an isothermal profile for the DM rather than a NFW distribution. The cored NIE is quite inadequate as a model of the DM so that galaxies, and in particular the BCG, have to supply additional convergence to broaden the central caustic. The discrepancy between the values of $L^{*}$ inferred from different hypotheses (see Tables 3 and 4), gives an estimate of the systematic uncertainties that plague galactic parameters inferred from lensing in our analysis.

Although a single NIE is unable to provide a good fit, when a second central peak associated with the BCG is added, the overall profile is no more isothermal. On the other hand a single NFW profile provides a good description of the overall mass distribution. However this advantage disappears when we focus on the cluster-sized dark matter distribution instead of the overall distribution. When we add separate components for the gas and the galactic halos, either an isothermal or a NFW profile for the dark matter provide a good fit. Exploiting Bayesian evidence should be the most accurate way to compare the two scenarios, but we are cautious of doing this for two main reasons. First, the reported evidences do not include a factor with depends on the priors on the cluster-sized halo parameters. Since we considered flat priors, the evidence depends on the allowed range. This a priori factor has no effect on the model comparison given a shape for the halo, but could affect the comparison between the isothermal and the NFW profile.

Furthermore, because of the large number of models and discontinuities, mainly associated with the central radial caustic, we had to perform our analysis in the source plane. Computation of the likelihood in the image plane for a number of models showed that the $\chi^{2}$ values in the source plane may be overestimated by $\gtrsim 2$ for the models with a NFW components, but be underestimated by $>2$ in the isothermal case.
Such effects can considerably affect any model comparison, so we prefer to address this problem in future work investigating a larger sample of clusters.

\section{Results}

We review the results that follow directly from our multicomponent parametric approach.

\subsection{Dynamical status}

The strong lensing analysis of the inner regions of AC 114 provides additional insight into its dynamical status. We have attempted a novel multiwavelength approach in which the baryonic components were mainly constrained using observations either in the X-ray or optical band, allowing us to infer directly the dark matter distribution from the lensing analysis. The gas was found to be systematically displaced from the dark matter. The main X-ray clump and the cluster-sized DM halo are off-centre by $\sim 9^{\prime \prime}$, an offset much larger than the Chandra accuracy of $\sim 1^{\prime \prime}$, which determines the accuracy of the X-ray peak position. The relative orientation differs by $(27 \pm 4) \mathrm{deg}$. On the other hand, the DM clump is nearly aligned with the X-ray tail. This implies that the X-ray surface brightness of the core has been strongly perturbed by the dynamical activity. The likely motion of a substructure toward the northeast, as suggested by the fronts, might have distorted the local emission causing a rotation of the overall surface brightness of the central X-ray clump towards the east and the relative misalignment of gas and dark matter.

We note that the above analysis is limited to the very inner regions probed by strong lensing. When averaged over larger scales, distribution features might differ and the impact of substructure should be properly addressed.

\subsection{Collisionless dark matter}

While the ICM is clearly displaced from the galaxy density, the dark matter distribution follows it. The quite large errors in the parameters describing either the number, luminosity, or stellar mass density distributions (see Table 1) make it difficult to determine whether a given galaxy density traces the dark matter distribution more closely than the others. However, the good agreement between each of them allows us to draw some conclusions. The galaxy and dark matter distributions have comparable centroid positions, orientations, and ellipticities. Since dark matter was modelled with a cusped profile, whereas the galaxy density was fitted with a cored distribution, the comparison cannot be extended to the remaining parameters. The agreement provides additional evidence of the collisionless nature of dark matter, as suggested by observations of the bullet cluster (Clowe et al. 2004). This time, we have been able to establish that the agreement between galaxies and dark matter concerns not only the location but also the shape of the distribution.

When comparing dark matter with the galaxy distributions, the agreement becomes striking when we consider the number density distribution. As aforementioned before, errors are quite large and definite statements cannot be drawn but the similarities between the expected values are nevertheless noteworthy. Galaxy abundance has been considered as a proxy of the cluster mass (Hicks et al. 2006). Our result provides additional evidence that galaxy number density is a dependable tracer also for DM shape and orientation. 


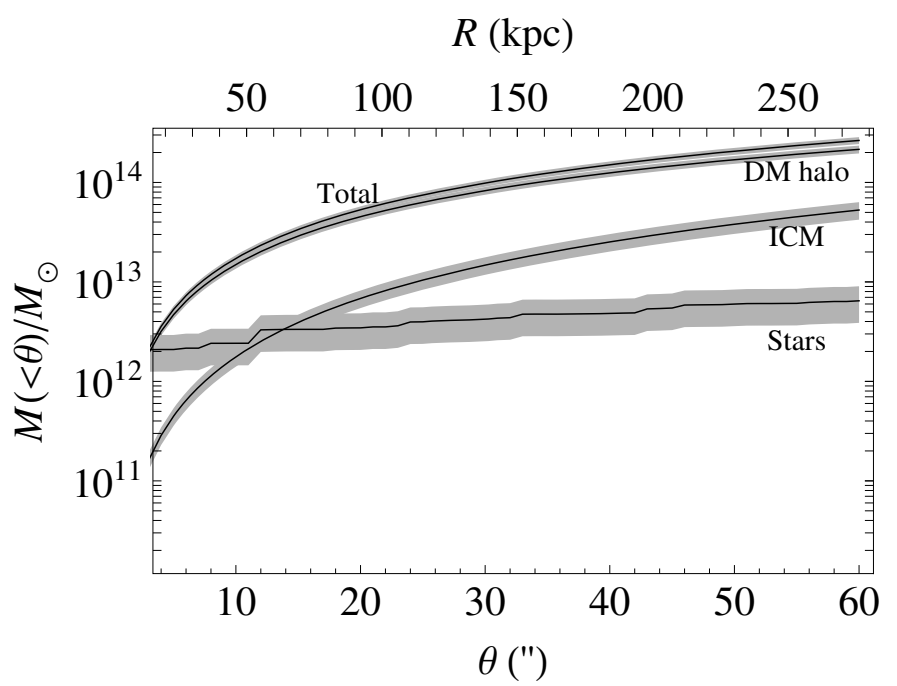

Fig. 5. Mass, in units of $M_{\odot}$, enclosed within a given projected radius for each component. Total mass, DM halo, ICM mass, and the stellar contribution from galaxies are plotted from the top to the bottom. DM halo refers to the cluster-sized dark matter component found in the multiwavelength lensing analysis.

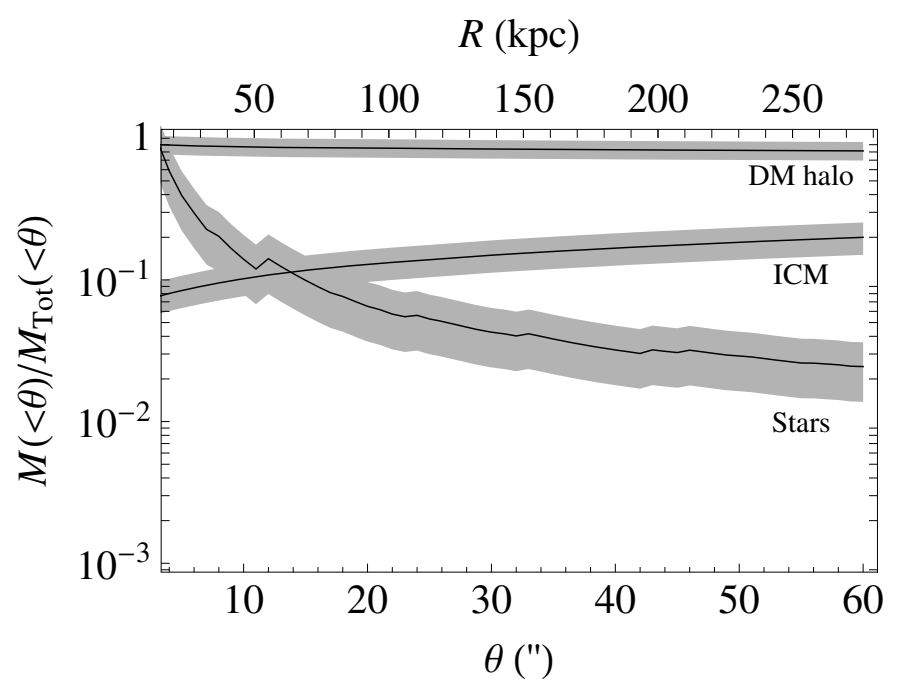

Fig. 6. Mass fractions as a function of the projected radius. Notation is the same as in Fig. 5.

\subsection{Baryons and dark matter}

Our multiwavelength approach allows us to determine the mass profile of each component in the very inner regions. Figures 5 and 6 show the enclosed projected masses within clustercentric distances smaller than $1^{\prime}(R \lesssim 280 \mathrm{kpc})$. We consider the two main baryonic components (stars in galaxies and ICM), the cluster-sized dark matter halo and the total projected mass (as modelled with a single NFW profile, see Sect. 6.1). The mass values with the smaller errors are those from the lensing analysis. The estimates of the different mass components scale differently with the Hubble constant, so that for comparison we fixed $h=0.7$. We note that we consider projected mass distributions for each component, which avoids biases caused by comparing projected with three-dimensional quantities. We recall that the mass of each component has been derived using a different method: the DM distribution has been inferred from lensing, whereas the ICM and the stellar mass have been estimated from X-ray data (see Sect. 4), and optical light data (see Sect. 2.1), respectively.

We found typical trends (Biviano \& Salucci 2006). The dark matter halo is the most prominent component $(\sim 80 \pm 10 \%$ at $R \sim 280 \mathrm{kpc})$. At the centre $(R \lesssim 50 \mathrm{kpc})$, the baryonic budget is dominated by the stellar mass of the BCG, whereas the ICM contribution is more important at larger radii. The gas distribution with radius is shallower than the dark matter profile, so that the ICM fraction increases from $\sim 10 \pm 3 \%$ at $R \sim 50 \mathrm{kpc}$ to $\sim 20 \pm 5 \%$ at $R \sim 280 \mathrm{kpc}$. These values are larger but still compatible with typical values inferred from X-ray analyses of luminous clusters (Allen et al. 2008). On the other hand, the stellar fraction is only a few percent at $R \gtrsim 150 \mathrm{kpc}$ (Biviano \& Salucci 2006).

The luminosity function of $\mathrm{AC} 114$ has been extensively studied (Andreon et al. 2005). Adopting a total luminosity of $(1.5 \pm 0.2) \times 10^{12} L_{\odot}$ in $r$ and $(1.9 \pm 1.2) \times 10^{11} L_{\odot}$ in $B$ within $0.6 \mathrm{Mpc} / h$ (Krick \& Bernstein 2007), we derive massto-light ratios of $M / L_{r}=(670 \pm 110) M_{\odot} / L_{\odot}$ and $M / L_{B}=$ (4400 \pm 1400$) M_{\odot} / L_{\odot}$, which are indicative of a underluminous cluster core. However, because of the large errors, especially in the $B$-band, the mass-to-light ratios remain slightly compatible with estimates from other clusters (Rines et al. 2004; Biviano 2008).

\section{Comparison with theoretical predictions}

It is of interest to compare the results of our analysis with the expectations of $\mathrm{N}$-body simulations or theoretical studies. The comparison requires an extrapolation of our mass model to larger radii, well beyond that directly probed by strong lensing. For this reason, we decided to limit the analysis in this section only to the global fits, i.e., to mass models that describe the total matter distribution (baryons and dark matter) within a single clustersized halo. The following considerations can then be seen as a corollary of the main result of the paper, i.e., that ICM has an effect in lens modelling, and do not make use of the explicit gas modelling discussed before.

\subsection{Inner slope}

Values of the inner and outer slopes of density profiles predicted by $N$-body simulations are still debated with different parameterizations competing (Merritt et al. 2006; Saha \& Read 2009). Baryons play a role too, since their infalling would steepen the dark matter profile. However, in large clusters this effect is expected to be small exterior to $\sim 20 \mathrm{kpc}$ (Gnedin et al. 2004). The general consensus is that in the inner regions of clusters the dark matter profile should go as $\rho \sim r^{-\alpha}$, where $\alpha$ is between 1 and 1.4 (Diemand et al. 2004).

To investigate the inner slope, we considered a total matter distribution modelled as a singular softened power-law $\left(r_{\mathrm{c}}=0\right)$, which represents a power law mass profile, i.e., $\rho \propto r^{-\alpha}$. This mass profile is able to reproduce all the images of the observed systems, and for the slope we obtained a best-fit model value of $\alpha=1.38 \pm 0.01$. This estimate is determined mainly by the images near the central radial caustic.

The simple power-law used in our analysis allow us to compare with previous analyses that employed the same parameterization, showing good agreement (Saha et al. 2006). On the other hand, the very small uncertainty in $\alpha$ is caused more by not enough accurate modelling than to very precise statistical accuracy. Since we were mainly interested in comparing with $N$ body simulations, we modelled the total mass profile as a single 
power-law. We did not attempt to distinguish a baryonic from a DM component. Inferring the inner slope of the DM component would require a far more detailed modelling (Limousin et al. 2008). Different parameterizations of the $\mathrm{cD}$ galaxies can produce an uncertainty of $\sim 0.05$ in the inner slope (Limousin et al. 2008). On the other hand, when the ICM mass distribution is modelled with a cored profile, the estimate of $\alpha$ does not depend on the inclusion of the gas in the fit procedure.

The main source of error $(\sim 0.1)$ is related to the absence of a length scale in the simple power-law profile we used. We can quantify the uncertainty with the following simple reasoning. The slope of a NFW profile changes from $\alpha=1$ in the very inner regions to $\alpha \simeq 1.18$ at $r \simeq 10^{-1} r_{\mathrm{s}}$, with a mean value of $\langle\alpha\rangle \simeq 1.12$. Then, for sets of multiple images covering nearly one tenth of the length scale, modelling the profile with a power-law causes an overestimate of the inner slope $\alpha$ of $\sim 0.1$. An additional source of error is caused by the degeneracy between the slope and the scale radius of a generalized NFW profile. Limousin et al. (2008) showed that fixing $r_{\mathrm{s}}$ to a value smaller that the best-fit model estimate causes an underestimate of the slope. The related uncertainty is $\sim 0.05$ (Limousin et al. 2008). Even after accounting for these systematics, we see that the estimated value of the inner slope of AC 114 remains steeper than a simple NFW profile and falls just in the middle of the range compatible with theoretical predictions (Diemand et al. 2004).

\subsection{Concentration}

The concentration parameter depends on the central density of the halo, uncovering imprints of the halo assembly history and thereby of its time of formation. When analysing ellipsoidal halos, we need generalized definitions for the intrinsic NFW parameters. We follow Corless \& King (2007), who defined a triaxial radius $r_{200}$ such that the mean density contained within an ellipsoid of semi-major axis $r_{200}$ is 200 times the critical density at the halo redshift; the corresponding concentration is $c_{200} \equiv r_{200} / r_{\mathrm{s}}$. The characteristic overdensity in terms of $c_{200}$ is then the same as that of a spherical profile. The mass, $M_{200}$, is the mass within the ellipsoid of semi-major axis $r_{200}$. The parameters $c_{200}$ and $M_{200}$ defined in this way have small deviations from the parameters computed by fitting spherically averaged density profiles, as performed in $N$-body simulations. The only caveat is that the spherical mass obtained in simulations is significantly less than the ellipsoidal $M_{200}$ for extreme axial ratios (Corless \& King 2007).

We estimated $M_{200}$ and $c_{200}$ from the projected NFW parameters directly inferred from the fit, i.e., $r_{\mathrm{sP}}$ and $k_{\mathrm{NFW}}$, see Appendix C. We assumed that a single NFW profile accurately describes the overall mass distribution (see Sect. 6.1). A main source of uncertainty is related to triaxiality issues (Gavazzi 2005; Oguri et al. 2005; Corless et al. 2009). A simple way of accounting for projection effects is detailed in Appendix C. The expected values of the geometrical correction factors can be estimated assuming random orientations and intrinsic axial ratios of probability density obtained from the results of $N$-body simulations (Jing \& Suto 2002). To compare our results with theoretical predictions, we did not consider a generalized NFW profile but we fixed the inner slope to $\alpha=1$, see Sect. 6.1. We obtained $c_{200}=3.5 \pm 0.7$, in close agreement with the estimate based on the velocity dispersion derived in Sect. 3.1, and $M_{200}=(1.3 \pm 0.9) \times 10^{15} M_{\odot} / h$, slightly lower than the mass from the virial theorem (see Sect. 3.1). We note that neglecting projection effects, the error on $c_{200}$ would have been $\sim 0.2$. We obtain nearly the same value for $c_{200}(=3.7 \pm 0.8)$ if we consider the cluster-sized DM halo instead of the total mass distribution. Because of the flatness of the ICM distribution, the central value for the concentration of the DM halo is higher than when we consider the overall distribution. However, the shift is smaller that the statistical uncertainty.

The halo concentration parameter is expected to be related to its virial mass, the concentration decreasing gradually with mass (Bullock et al. 2001). According to some recent numerical simulations (Duffy et al. 2008), the concentration of a cluster with the same mass as that just derived for AC 114 at its redshift should be $c_{200}=2.90 \pm 0.13 \pm 0.13$. The agreement with our result is striking, something unusual when comparing concentrations derived from lensing analyses to predicted values.

\section{Discussion}

Multiwavelength analyses of lensing observations are a very powerful tool to constrain properties of galaxy clusters (Fox \& Pen 2002; Clowe et al. 2004; Smith et al. 2005; Sereno 2007; Lemze et al. 2009). Here, we have performed a lensing analysis in which the gas mass distribution, previously inferred from $\mathrm{X}$-ray observations, has been included from the very beginning in the modelling. Gas is the main baryonic component and typically contributes $>10 \%$ of the total mass of galaxy clusters (Allen et al. 2008). Considering the ICM in the parameterization can be see as an improvement on the usual way of modelling only cluster-sized dark matter and galaxy-sized halos. We have attempted a first step in this direction.

The main result of this paper is that the explicit inclusion of the gas distribution has an important effect on lensing modelling. While it is well known that galactic halos have to be accounted for to increase the accuracy of strong lensing modelling, we have demonstrated that including an additional component that accounts for the gas distribution also helps. Models in which the cluster-sized dark matter halo is considered with the ICM provide more accurate results than parametric solutions with a single halo that accounts at the same time for both dark matter and diluted baryons. The physical gain is quite significant too, since modelling the gas on its own allows us to place direct observational constraints on the dark matter distribution. This is crucial to the understanding of the formation of cosmic structures and the co-evolution of baryons and dark matter in clusters of galaxies.

X-ray data are usually exploited to investigate the mass distribution of a galaxy cluster on a larger scale than the very inner regions analysed with strong lensing analyses. However, X-ray telescopes also map the intracluster medium in the central parts. Our method has been designed to exploit this information. We do not rely on the usual hypotheses needed to infer the total mass from X-ray data (e.g., hydrostatic equilibrium and/or constant baryonic fraction) which are more accurate within a radius as large as the virial one. We only consider the gas distribution directly measured from X-ray observations, which is reliable on all scales. As far as a study of the inner regions is concerned, no calibration on a larger scale, such as those provided by weaklensing studies, is then needed.

As a test-bed, we have applied our novel method to AC 114, a dynamically active cluster. Comparison of the dark matter map directly obtained from lensing modelling with either the gas or the stellar mass distribution can provide important insight into the properties of the cluster. Our analysis of AC 114 identified another example in which the ICM is displaced from the dark 
matter in dynamically active clusters, while the collisionless nature of dark matter was confirmed by the close matching with the galaxy distribution.

Our lensing analysis provided a mass modelling of AC 114 that can be compared with $N$-body simulations. The obtained results are in remarkable agreement with predictions. For AC 114, we found that: i) a cusped NFW model for the overall mass distribution seems to be preferred over an isothermal profile; ii) the inner slope is slightly steeper than a simple NFW; iii) the concentration parameter is in line with predictions from massconcentration scaling relations.

However, comparisons with $N$-body simulations must be taken cum grano salis. Statistical samples of halos with the mass of a galaxy cluster are very demanding to obtain with numerical simulations. In addition, AC 114 is dynamically active, which makes the comparison even more ambiguous. Furthermore, our estimation of the virial mass and of the concentration parameter required an extrapolation to scales much larger than what mapped by strong lensing. Calibration with other methods, such as weak lensing, is then needed to support conclusions on the global properties. Nevertheless, we feel encouraged by the agreement between the parameters estimated with lensing and those inferred by a dynamical analysis.

Our estimated inner slope agrees with the estimates of numerical simulations. A value of $\alpha \gtrsim 1$ might be an indication of steepening caused by adiabatic contraction, but the very young dynamical age of AC 114 and its intense ongoing merging activity weaken this interpretation. We note that the modelling of the seemingly relaxed cluster A 1703 fitted an inner slope larger than one ( $\alpha \sim 1.1)$ too (Limousin et al. 2008).

Different models used for the BCG do not change our results. For a comparison with numerical simulations, we used global fits (i.e., a single DM+baryon halo); as far as the comparison between DM and gas (or light) is concerned, different assumptions about the BCG do not significantly affect either the orientation or centroid of the DM distribution. Finally, as far as the integrated mass distributions of the different components are concerned, the stellar mass estimate is based solely on measured photometry, whereas the gas mass is derived from X-ray observations.

Lensing clusters appear to be quite over-concentrated (Comerford \& Natarajan 2007; Johnston et al. 2007; Broadhurst et al. 2008; Mandelbaum et al. 2008; Oguri \& Blandford 2009; Oguri et al. 2009; Okabe et al. 2010; Corless et al. 2009). The analysis that we have performed provides additional insights. First, some peculiarities ensure that our results are less affected by biases. We derived the concentration parameter using only strong lensing data and we did not use the spherical approximation of the halo profile. Different definitions of parameters for spherically averaged profiles can play a role when comparing observations to predictions (Broadhurst \& Barkana 2008). Second, AC 114 has some peculiar features that might make the high concentration problem much less pronounced. In particular, the very long tail in the X-ray morphology and the detection of a shock front suggest that the cluster develops in the plane of sky. The elongation of the cluster could be probed by observationally combining lensing and X-ray data with measurements of the Sunyaev-Zeldovich effect (Fox \& Pen 2002; De Filippis et al. 2005; Sereno et al. 2006; Sereno 2007). Unfortunately, detection for AC 114 is still marginal (Andreani et al. 1996) and deeper radio observations are needed.

Acknowledgements. The authors thank E. De Filippis and P. Martini for some useful clarifications. For the first stages of this work, M.S. has been supported by the Swiss National Science Foundation.

\section{Appendix A: Velocity dispersion}

A cluster galaxy velocity dispersion represents a crucial source of information. We collected positions and redshifts of galaxies in the vicinity of AC 114 from the NASA/IPAC Extragalactic

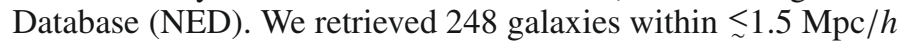
from the $\mathrm{BCG}$ in the redshift range $0.1 \leq z \leq 0.5$. A careful treatment of interlopers is required in dynamical modelling. Many approaches have been proposed and their efficiency has been tested using numerical simulations (Wojtak et al. 2007; Biviano et al. 2006). Here, we propose a method of interloper removal that combines several approaches.

To select member galaxies, we first exploit velocity information using an adaptive kernel technique (Pisani 1993, 1996). This nonparametric method evaluates the underlying density probability function from the observed discrete data-set. We identify the main peak in the distribution and reject galaxies that do not belong to this peak. This cut has been successfully employed in many studies (Girardi et al. 1998; Girardi \& Mezzetti 2001). To evaluate the optimal smoothing parameter, we minimise the integrated square error (Pisani 1993), fixing the initial value to the estimate proposed by Vio et al. (1994). As a second step, we take into account both the position and the velocity information by using the procedure of the shifting gapper (Fadda et al. 1996; Girardi et al. 1998). This method combines velocity information with the clustercentric radial distance. In each bin, shifting along the radial distance form the centre, a galaxy is removed if separated from the main local body by more than a fixed gap in velocity. We use a gap of $\geq 1000 \mathrm{~km} \mathrm{~s}^{-1}$ in the cluster rest frame and a bin of $0.3 / h \mathrm{Mpc}$.

As a third and final step, we employ a Bayesian technique (Andreon et al. 2008). The effect of a contaminating population can be inferred by considering that data $v_{i}$ come from a Gaussian-distributed intensity superimposed on an homogeneous random process (van der Marel et al. 2000; Mahdavi \& Geller 2004; Andreon et al. 2008)

$p\left(v_{i} ; f_{\mathrm{cl}}, v_{\mathrm{cl}}, \sigma_{\mathrm{los}}, \Delta v\right)=f_{\mathrm{cl}} \mathcal{N}\left(v_{\mathrm{cl}}, \sigma_{\mathrm{los}}\right)+\left(1-f_{\mathrm{cl}}\right) / \Delta v$,

where $f_{\mathrm{cl}}$ is the fraction of member galaxies, $\mathcal{N}$ is a normal distribution centred on $v_{\mathrm{cl}}$ with dispersion $\sigma_{\mathrm{los}}$, and $\Delta v$ is the velocity range spanned by data. The likelihood is then given by

$\mathcal{L} \propto \prod_{i} p\left(v_{i} ; \ldots\right)$

After considering a sharp prior on $\Delta v=\max \left\{v_{i}\right\}-\min \left\{v_{i}\right\}$ and flat priors on the other parameters, we derive the final probability. This powerful statistical method, which estimated the total fraction of interlopers without actually identifying them, has been employed to constrain the phase-space probability function (van der Marel et al. 2000; Mahdavi \& Geller 2004), but is rarely used to constrain the velocity dispersion (Andreon et al. 2008). After marginalization, we obtain estimates of $z_{\mathrm{cl}}=$ $0.3153 \pm 0.0007$ for the cluster mean redshift and $\sigma_{\mathrm{los}}=1900 \pm$ $100 \mathrm{~km} \mathrm{~s}^{-1}$ for the velocity dispersion, the estimated fraction of interlopers being $(11 \pm 6) \%$, in good agreement with the estimates from numerical simulations that showed that using nonBayesian methods, $\sim 18 \%$ of selected members are unrecognized interlopers (Biviano et al. 2006). Standard corrections for cosmological effects and velocity errors $\left(\delta v \sim 150 \mathrm{~km} \mathrm{~s}^{-1}\right)$ are applied (Danese et al. 1980). Our final estimates of $z$ and $\sigma_{\text {los }}$ are remarkably stable for different thresholds and gaps in the first steps of our selection procedure. Only the estimated fraction of non-members is sensitive to the details of the previous cuts. The 
results of the Bayesian technique just employed are also stable in the case of an intrinsically skewed velocity distribution (Andreon et al. 2008).

Our expected value for $\sigma_{\text {los }}$ is lower (but compatible within the errors) than the estimate of Martini et al. (2007), but higher, despite being marginally compatible, with early estimates from Couch \& Sharples (1987) and Mahdavi \& Geller (2001). On the other hand, the estimate of Girardi \& Mezzetti (2001), who considered only a sample of non-active galaxies, is substantially lower.

\section{Appendix B: Substructures}

Substructures in the galaxy distributions of AC 114 have been detected with several tecniques. Two clumps of galaxies, the first one northwest (NW clump) and the second one southest (SE clump) of the BCG were noted by Natarajan et al. (1998), who combined optical data with weak lensing. According to a combined weak and strong lensing modelling (Campusano et al. $2001)$, the NW clump at $\left\{\theta_{1}, \theta_{2}\right\} \sim\left\{80^{\prime \prime}, 30^{\prime \prime}\right\}$ and the SE clump at $\left\{-75^{\prime \prime},-75^{\prime \prime}\right\}$ have a mass $\sim 20 \%$ and $\sim 35 \%$, respectively, of the mass of the main clump associated with the central cD galaxy. A clump of galaxies resembling a group with its own cD-like galaxy at $\sim 1.1 \mathrm{Mpc} / h$ northwest of the BCG was noted by Krick \& Bernstein (2007), who also found evidence of associated intracluster light emission. Actually, the main galaxy in the NW clump is as luminous as the central BCG galaxy (Couch et al. 1998; Stanford et al. 2002). The luminosity map also reveals other features, most notably a filament towards the northeast in the very inner region, almost perpendicular to the overall orientation.

By combining galaxy velocity and position information, we can identify local substructures and compact subsystems. Here, we wish to use this information fro this purpose. The $\Delta_{\mathrm{DS}}$ test (Dressler \& Shectman 1988) searches for significant deviations in local groups that have either an average velocity $\bar{v}_{\text {loc }}$ that differs from the cluster mean, $\bar{v}_{\text {glo }}$, or a velocity dispersion, $\sigma_{\text {loc }}$, that differs from the global one, $\sigma_{\text {glo }}$. For our analysis, we considered the classic version of the test, which considers all possible subgroups of ten neighbors around each cluster galaxy (Dressler \& Shectman 1988) with the only slight difference being that to calculate location and scale we consider the biweight estimators instead of mean and standard deviation (Beers et al. 1990). The deviation for each galaxy can then be expressed as (Dressler \& Shectman 1988)

$\delta_{\mathrm{DS}}^{2}=\frac{11}{\sigma_{\mathrm{glo}}^{2}}\left[\left(\bar{v}_{\mathrm{loc}}-\bar{v}_{\mathrm{glo}}\right)^{2}+\left(\sigma_{\mathrm{loc}}-\sigma_{\mathrm{glo}}\right)^{2}\right]$.

The parameter $\Delta_{\mathrm{DS}}=\sum \delta_{\mathrm{DS}}$ quantifies the overall presence of substructures. The $\Delta_{\mathrm{DS}}$ statistic is then obtained by comparing the measured value with those obtained from simulated samples generated randomly shuffling velocities. The probability $P_{\Delta_{\mathrm{DS}}}$ that the observed value is caused by noise is then given by the fraction of samples with a value of $\Delta_{\mathrm{DS}}$ larger than the observed one. We get $P_{\Delta \mathrm{Ds}} \lesssim 0.1$. We also considered modified versions of the test (Biviano et al. 2002) but our results were unaffected.

The method can be adapted to determine which galaxies have the highest likelihood of residing in subclusters. This can be achieved by considering the $\delta_{\mathrm{DS}}$-statistic in a way similar to what we have just done for $\Delta_{\mathrm{DS}}$. The $\delta_{\mathrm{DS}}$-analysis identifies a potential substructure $320^{\prime \prime}(\sim 1.0 \mathrm{Mpc} / h)$ northeast of the BCG. Four galaxies located at $\left\{\theta_{1}, \theta_{2}\right\} \sim\left\{-200^{\prime \prime}, 250^{\prime \prime}\right\}$ $(\sim\{-0.64,0.80\} \mathrm{Mpc} / h)$ have a chance in excess of $99.8 \%$ of belonging to a substructure. In conclusion, the $\Delta_{\mathrm{DS}}$ and the $\delta_{\mathrm{DS}}$-test provide additional evidence for dynamical activity.

\section{Appendix C: Projection effects}

The projected map $F_{2 \mathrm{D}}$ of a volume density $F_{3 \mathrm{D}}$, which is constant on surfaces of constant ellipsodial radius $\zeta$, is elliptical on the plane of the sky (Stark 1977; Sereno 2007)

$F_{2 \mathrm{D}}\left(\xi ; l_{\mathrm{P}}, p_{i}\right)=\frac{2}{\sqrt{f}} \int_{\xi}^{\infty} F_{3 \mathrm{D}}\left(\zeta ; l_{\mathrm{s}}, p_{i}\right) \frac{\zeta}{\sqrt{\zeta^{2}-\xi^{2}}} \mathrm{~d} \zeta$

where $\xi$ is the elliptical radius in the plane of the sky, $l_{\mathrm{s}}$ is the typical length scale of the $3 \mathrm{D}$ density, $l_{\mathrm{P}}$ is its projection on the plane of the sky, and $p_{i}$ are the other parameters describing the intrinsic density profile (slope, ...); the subscript $\mathrm{P}$ denotes measurable projected quantities. The parameter $f$ depends on the intrinsic shape and orientation of the 3D distribution

$f=e_{1}^{2} \sin ^{2} \theta_{\mathrm{Eu}} \sin ^{2} \varphi_{\mathrm{Eu}}+e_{2}^{2} \sin ^{2} \theta_{\mathrm{Eu}} \cos ^{2} \varphi_{\mathrm{Eu}}+\cos ^{2} \theta_{\mathrm{Eu}}$,

where $\varphi_{\mathrm{Eu}}, \theta_{\mathrm{Eu}}$ are the two Euler's angles of the principal cluster axes that fix the orientation of the line of sight, and $e_{i}(\geq 1), i=$ $\{1,2\}$ are the two intrinsic axial ratios (Sereno 2007). The integral in Eq. (C.1) is proportional to $l_{\mathrm{s}}$. The relation between a length measured along the major axis and its projection in the sky is

$\frac{l_{\mathrm{s}}}{\sqrt{f}} \equiv \frac{l_{\mathrm{P}}}{e_{\Delta}}$,

where the parameter $e_{\Delta}$ quantifies the elongation of the triaxial ellipsoid along the line of sight (Sereno 2007),

$e_{\Delta}=\left(\frac{e_{\mathrm{P}}}{e_{1} e_{2}}\right)^{1 / 2} f^{3 / 4}$

where $e_{\mathrm{P}}(\geq 1)$ is the projected axial ratio. According to our notation for the NFW profile, the intrinsic $l_{\mathrm{s}}$ and the projected $l_{\mathrm{P}}$ have to be read as $r_{\mathrm{s}}$ and $r_{\mathrm{sP}}$, respectively. Finally, the surface density can be expressed as

$F_{2 \mathrm{D}}=\frac{l_{\mathrm{P}}}{e_{\Delta}} f_{2 \mathrm{D}}\left(\xi ; l_{\mathrm{P}}, p_{i}, \ldots\right)$,

where $f_{2 \mathrm{D}}$ has the same functional form as a spherically symmetric halo. When we deproject a surface density, the normalization of the volume density can then be known only to within a geometrical factor

$f_{\mathrm{geo}} \equiv \frac{\left(e_{1} e_{2}\right)^{1 / 2}}{f^{3 / 4}}=\frac{e_{\mathrm{P}}^{1 / 2}}{e_{\Delta}}$.

When we estimate the gas mass from measurements of the surface brightness $S_{\mathrm{X}}$, which is proportional to the squared density $n_{\mathrm{e}}^{2}$, we have first to deproject $S_{X}$, so that, after inversion, the only unknown in the expression of the central density is the factor $f_{\text {geo }}^{-1 / 2}$. We then project the density $n_{\mathrm{e}}$ along the line of sight, which brings about an additional factor $f_{\text {geo }}$. The resulting central projected mass density is $\Sigma_{0} \propto f_{\mathrm{geo}}^{1 / 2}$. This geometrical factor is independent of the specific density profile of the ICM distribution.

When inferring the concentration parameter of the matter distribution, we face a slightly different case. We have just a single projection, so that the central convergence of a NFW profile 
estimated from lensing can be written in terms of $c_{200}$ and the projected scale-length modulus a factor $f_{\text {geo }}$,

$\Sigma_{\mathrm{cr}} \times \kappa_{\mathrm{NFW}}=\frac{f_{\text {geo }}}{\sqrt{e_{\mathrm{P}}}} \rho_{\mathrm{s}} r_{\mathrm{sP}}$.

The estimate of the mass $M_{200}$ also depends on the scale-length $r_{\mathrm{s}}$, and in turn on the unknown factor $\sqrt{f} / e_{\Delta}$ (see Eq. (C.3)). Then

$M_{200}=\frac{4 \pi}{3} \times 200 \rho_{\mathrm{cr}} \times\left(c_{200} r_{\mathrm{sP}}\right)^{3} \frac{f_{\mathrm{geo}}}{e_{\mathrm{P}}^{3 / 2}}$,

where $\rho_{\mathrm{cr}}$ is the critical density of the universe at the cluster redshift.

The geometrical correction factors can be estimated based on some working hypotheses. Assuming that the cluster is drawn from a population with random orientations and intrinsic axial ratios with probability density following results from $N$-body simulations (Jing \& Suto 2002), we can estimate for $f_{\text {geo }}\left(f_{\text {geo }}^{1 / 2}\right)$ an expected value of $0.93(0.95)$ with a dispersion of $0.37(0.18)$. The concentration is obtained from Eq. (C.7) after expressing $\rho_{\mathrm{s}}$ in terms of $c_{200}$ for an ellipsoidal halo (Corless \& King 2007).

\section{References}

Allen, S. W., Rapetti, D. A., Schmidt, R. W., et al. 2008, MNRAS, 383, 879 Andreani, P., Pizzo, L., dall'Oglio, G., et al. 1996, ApJ, 459, L49 Andreon, S., de Propris, R., Puddu, E., Giordano, L., \& Quintana, H. 2008, MNRAS, 383, 102

Andreon, S., Punzi, G., \& Grado, A. 2005, MNRAS, 360, 727

Beers, T. C., Flynn, K., \& Gebhardt, K. 1990, AJ, 100, 32

Bell, E. F., \& de Jong, R. S. 2001, ApJ, 550, 212

Binney, J., \& Merrifield, M. 1998, Galactic astronomy (Princeton, NJ: Princeton University Press)

Binney, J., \& Tremaine, S. 1987, Galactic dynamics (Princeton, NJ: Princeton University Press)

Biviano, A. 2008, unpublished [arXiv: 0811.3535]

Biviano, A., \& Salucci, P. 2006, A\&A, 452, 75

Biviano, A., Katgert, P., Thomas, T., \& Adami, C. 2002, A\&A, 387, 8

Biviano, A., Murante, G., Borgani, S., et al. 2006, A\&A, 456, 23

Bradač, M., Allen, S. W., Treu, T., et al. 2008, ApJ, 687, 959

Brainerd, T. G., Blandford, R. D., \& Smail, I. 1996, ApJ, 466, 623

Broadhurst, T., Umetsu, K., Medezinski, E., Oguri, M., \& Rephaeli, Y. 2008, ApJ, 685, L9

Broadhurst, T. J., \& Barkana, R. 2008, MNRAS, 390, 1647

Bullock, J. S., Kolatt, T. S., Sigad, Y., et al. 2001, MNRAS, 321, 559

Campusano, L. E., Pelló, R., Kneib, J.-P., et al. 2001, A\&A, 378, 394

Clowe, D., Gonzalez, A., \& Markevitch, M. 2004, ApJ, 604, 596

Cole, S., Norberg, P., Baugh, C. M., et al. 2001, MNRAS, 326, 255

Comerford, J. M., \& Natarajan, P. 2007, MNRAS, 379, 190

Corless, V. L., \& King, L. J. 2007, MNRAS, 380, 149

Corless, V. L., King, L. J., \& Clowe, D. 2009, MNRAS, 393, 1235

Couch, W. J., \& Sharples, R. M. 1987, MNRAS, 229, 423

Couch, W. J., Balogh, M. L., Bower, R. G., et al. 2001, ApJ, 549, 820

Couch, W. J., Barger, A. J., Smail, I., Ellis, R. S., \& Sharples, R. M. 1998, ApJ, 497, 188

Danese, L., de Zotti, G., \& di Tullio, G. 1980, A\&A, 82, 322

De Filippis, E., Bautz, M. W., Sereno, M., \& Garmire, G. P. 2004, ApJ, 611, 164

De Filippis, E., Sereno, M., Bautz, M. W., \& Longo, G. 2005, ApJ, 625, 108

Diemand, J., Moore, B., \& Stadel, J. 2004, MNRAS, 353, 624

Dressler, A., \& Shectman, S. A. 1988, AJ, 95, 985
Duffy, A. R., Schaye, J., Kay, S. T., \& Dalla Vecchia, C. 2008, MNRAS, 390, L64

Fadda, D., Girardi, M., Giuricin, G., Mardirossian, F., \& Mezzetti, M. 1996, ApJ, 473,670

Fox, D. C., \& Pen, U.-L. 2002, ApJ, 574, 38

Gavazzi, R. 2005, A\&A, 443, 793

Gerhard, O., Kronawitter, A., Saglia, R. P., \& Bender, R. 2001, AJ, 121, 1936

Girardi, M., \& Mezzetti, M. 2001, ApJ, 548, 79

Girardi, M., Giuricin, G., Mardirossian, F., Mezzetti, M., \& Boschin, W. 1998, ApJ, 505, 74

Gnedin, O. Y., Kravtsov, A. V., Klypin, A. A., \& Nagai, D. 2004, ApJ, 616, 16

Hennawi, J. F., Dalal, N., Bode, P., \& Ostriker, J. P. 2007, ApJ, 654, 714

Hicks, A. K., Ellingson, E., Hoekstra, H., \& Yee, H. K. C. 2006, ApJ, 652, 232 Jing, Y. P., \& Suto, Y. 2002, ApJ, 574, 538

Johnston, D. E., Sheldon, E. S., Wechsler, R. H., et al. 2007, unpublished [arXiv: 0709.1159]

Keeton, C. R. 2001a, unpublished [arXiv:astro-ph/0102341]

Keeton, C. R. 2001b, unpublished [arXiv:astro-ph/0102340]

Krick, J. E., \& Bernstein, R. A. 2007, AJ, 134, 466

Lemze, D., Broadhurst, T., Rephaeli, Y., Barkana, R., \& Umetsu, K. 2009, ApJ, 701,1336

Limousin, M., Richard, J., Kneib, J.-P., et al. 2008, A\&A, 489, 23

Lin, Y.-T., Mohr, J. J., \& Stanford, S. A. 2003, ApJ, 591, 749

Mackay, D. J. C. 2003, Information Theory, Inference and Learning Algorithms (Cambridge Univ. Press, Cambridge)

Mahdavi, A., \& Geller, M. J. 2001, ApJ, 554, L129

Mahdavi, A., \& Geller, M. J. 2004, ApJ, 607, 202

Mandelbaum, R., Seljak, U., \& Hirata, C. M. 2008, JCAP, 8, 6

Mannucci, F., Basile, F., Poggianti, B. M., et al. 2001, MNRAS, 326, 745

Martini, P., Mulchaey, J. S., \& Kelson, D. D. 2007, ApJ, 664, 761

Merritt, D., Graham, A. W., Moore, B., Diemand, J., \& Terzić, B. 2006, AJ, 132 , 2685

Miranda, M., Sereno, M., de Filippis, E., \& Paolillo, M. 2008, MNRAS, 385, 511

Natarajan, P., Kneib, J.-P., Smail, I., \& Ellis, R. S. 1998, ApJ, 499, 600

Natarajan, P., Kneib, J., Smail, I., et al. 2009, ApJ, 693, 970

Navarro, J. F., Frenk, C. S., \& White, S. D. M. 1996, ApJ, 462, 563

Navarro, J. F., Frenk, C. S., \& White, S. D. M. 1997, ApJ, 490, 493

Oguri, M., \& Blandford, R. D. 2009, MNRAS, 392, 930

Oguri, M., Hennawi, J. F., Gladders, M. D., et al. 2009, ApJ, 699, 1038

Oguri, M., Takada, M., Umetsu, K., \& Broadhurst, T. 2005, ApJ, 632, 841

Okabe, N., Takada, M., Umetsu, K., Futamase, T., \& Smith, G. P. 2010, PASJ, 62,811

Okabe, N., \& Umetsu, K. 2008, PASJ, 60, 345

Pisani, A. 1993, MNRAS, 265, 706

Pisani, A. 1996, MNRAS, 278, 697

Rines, K., Geller, M. J., Diaferio, A., Kurtz, M. J., \& Jarrett, T. H. 2004, AJ, 128 , 1078

Rykoff, E. S., McKay, T. A., Becker, M. R., et al. 2008, ApJ, 675, 1106

Saha, P., \& Read, J. I. 2009, ApJ, 690, 154

Saha, P., Read, J. I., \& Williams, L. L. R. 2006, ApJ, 652, L5

Saha, P., Williams, L. L. R., \& Ferreras, I. 2007, ApJ, 663, 29

Sand, D. J., Treu, T., Ellis, R. S., Smith, G. P., \& Kneib, J.-P. 2008, ApJ, 674, 711

Schlegel, D. J., Finkbeiner, D. P., \& Davis, M. 1998, ApJ, 500, 525

Sereno, M. 2007, MNRAS, 380, 1207

Sereno, M., De Filippis, E., Longo, G., \& Bautz, M. W. 2006, ApJ, 645, 170

Smail, I., Ellis, R. S., Fitchett, M. J., et al. 1991, MNRAS, 252, 19

Smail, I., Couch, W. J., Ellis, R. S., \& Sharples, R. M. 1995, ApJ, 440, 501

Smith, G. P., Smail, I., Kneib, J.-P., et al. 2002, MNRAS, 330, 1

Smith, G. P., Kneib, J.-P., Smail, I., et al. 2005, MNRAS, 359, 417

Stanford, S. A., Eisenhardt, P. R., Dickinson, M., Holden, B. P., \& De Propris, R. 2002, ApJS, 142, 153

Stark, A. A. 1977, ApJ, 213, 368

van der Marel, R. P., Magorrian, J., Carlberg, R. G., Yee, H. K. C., \& Ellingson, E. 2000, AJ, 119, 2038

Vio, R., Fasano, G., Lazzarin, M., \& Lessi, O. 1994, A\&A, 289, 640

Wojtak, R., Łokas, E. L., Mamon, G. A., et al. 2007, A\&A, 466, 437 